\title{
Highly Accurate Attitude Estimation via Horizon Detection
}

Bertil Grelsson, Michael Felsberg and Folke Isaksson

The self-archived postprint version of this journal article is available at Linköping University Institutional Repository (DiVA):

http://urn.kb.se/resolve?urn=urn:nbn:se:liu:diva-108212

N.B.: When citing this work, cite the original publication.

Grelsson, B., Felsberg, M., Isaksson, F., (2016), Highly Accurate Attitude Estimation via Horizon Detection, Journal of Field Robotics, 33(7), 967-993. https://doi.org/10.1002/rob.21639

Original publication available at:

https://doi.org/10.1002/rob.21639

Copyright: Wiley (12 months)

http://eu.wiley.com/WileyCDA/ 


\title{
Highly accurate attitude estimation via horizon detection
}

\author{
Bertil Grelsson* \\ Saab Dynamics \\ Saab, Linköping, Sweden \\ bertil.grelsson@saabgroup.com
}

\author{
Michael Felsberg \\ Computer Vision Laboratory \\ Linköping University, Sweden \\ michael.felsberg@liu.se
}

\author{
Folke Isaksson \\ Vricon Systems \\ Saab, Linköping, Sweden \\ folke. isaksson@saabgroup.com
}

\begin{abstract}
Attitude (pitch and roll angle) estimation from visual information is necessary for GPS free navigation of airborne vehicles. We propose a highly accurate method to estimate the attitude by horizon detection in fisheye images. A Canny edge detector and a probabilistic Hough voting scheme are used to compute an approximate attitude and the corresponding horizon line in the image. Horizon edge pixels are extracted in a band close to the approximate horizon line. The attitude estimates are refined through registration of the extracted edge pixels with the geometrical horizon from a digital elevation map (DEM), in our case the SRTM3 database. The proposed method has been evaluated using 1629 images from a flight trial with flight altitudes up to $600 \mathrm{~m}$ in an area with ground elevations ranging from sea level up to $500 \mathrm{~m}$. Compared with the ground truth from a filtered IMU/GPS solution, the standard deviation for the pitch and roll angle errors are $0.04^{\circ}$ and $0.05^{\circ}$, respectively, with mean errors smaller than $0.02^{\circ}$. To achieve the high accuracy attitude estimates, the ray refraction in the earth atmosphere has been taken into account. The attitude errors obtained on real images are less or equal to those achieved on synthetic images for previous methods with DEM refinement, and the errors are about one order of magnitude smaller than for any previous vision-based method without DEM refinement.
\end{abstract}

\section{Introduction}

For autonomous navigation of unmanned aerial vehicles (UAVs), online estimation of the absolute position and attitude of the vehicle is required. For attitude estimation, inertial measurement units (IMUs) are commonly used but they suffer from drift and need support to give reliable estimates over time. Pilots in manned aircraft intuitively use the horizon as an attitude reference. Using the same basic concept, many vision-based methods have been presented where horizon detection is employed for absolute attitude estimates. Most of these methods include either an explicit or implicit assumption that the horizon line is smooth, (Natraj et al., 2013), (Mondragon et al., 2010), (Moore et al., 2011b), (Grelsson and Felsberg, 2013), (Bao et al., 2003), (Dusha et al., 2011) and (Dumble and Gibbens, 2012). In this context, we define the

\footnotetext{
*Alternative address: Computer Vision Laboratory, Linköping University, Sweden. E-mail bertil.grelsson@liu.se
} 


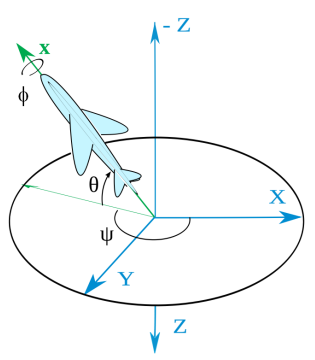

(a)
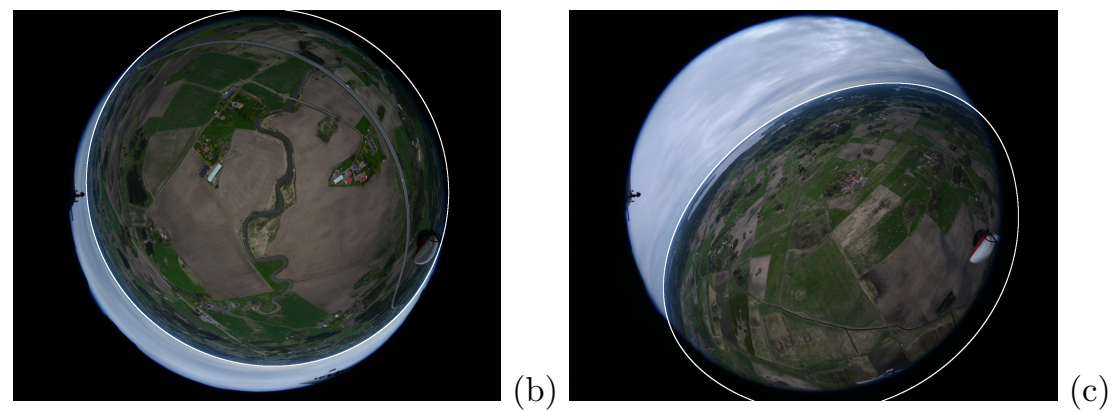

Figure 1: (a) Definition of vehicle pose, $\psi=$ yaw, $\theta=$ pitch, $\phi=$ roll. (b), (c) Aerial fisheye images with estimated horizon overlaid.

horizon as the boundary line between the sky and the ground/sea pixels in the image. The cited methods often search for or approximate the horizon as straight lines in perspective images or perfect ellipses in omnidirectional images. The attitude accuracy obtained with these methods is often around $1-2^{\circ}$. This accuracy is sufficient if your aim is to control and stabilize a UAV during flight, even for rather extreme maneuvers (Thurrowgood et al., 2010). But for autonomous surveillance and reconnaissance missions with flight durations around an hour covering distances in the order of $100 \mathrm{~km}$, this attitude accuracy is far from adequate. The position estimates would drift away and hence the UAV would deviate too far from its intended route implying that the estimated position for localized objects of interest in the images would be very inaccurate. A constant $1^{\circ}$ attitude error for a $100 \mathrm{~km}$ flight results in a position error around $2 \mathrm{~km}$. For these types of missions absolute attitude accuracies better than $0.1^{\circ}$ are often required throughout the flight.

In a recent paper (Dumble and Gibbens, 2014), a method is proposed where the measured $360^{\circ}$ horizon profile from a set of aerial perspective images is aligned with a reference horizon profile computed from a digital elevation model (DEM) to estimate the attitude and the position of the platform. This is the only method we have encountered that can provide these high accuracy attitude estimates However, as will be discussed later, this method requires a large horizon profile variance and that the distance to the perceived horizon is relatively short $(<40 \mathrm{~km})$. This makes the method ideal for, but also restricted to, mountainous terrain like the Alps, where the method was evaluated.

Mountainous terrain, with ground elevations greater than $2 \mathrm{~km}$, accounts for about $4 \%$ of the total area on earth (Encyclopaedia Britannica, 2014). Terrain with ground elevations lower than one kilometer is by far the most common land type on earth covering more than $20 \%$ of the total area and around $70 \%$ of the total land area on earth. Moreover, urban areas account for only $3 \%$ of the total land area on earth (NewGeography, 2010). Attitude estimation methods for low altitude flights in urban environment have been adressed in e.g. (Bazin et al., 2008) and (Demonceaux et al., 2007). Our aim is to develop a method that provides highly accurate attitude estimates in the most common terrain type, i.e. flying above tree tops and buildings in terrain with ground elevations up to a kilometer.

We present a novel method for highly accurate estimation of the pitch and roll angles of an airborne vehicle via horizon detection in fisheye images, see figure 1(a) for definitions of angles. We use 30 MPixel images with a frame rate of around $1 \mathrm{fps}$. The attitude estimates are refined through registration of the perceived horizon with the geometrical horizon from a DEM. To the best of our knowledge, this is the first proposed method for aerial omnidirectional images where the detected horizon is refined with DEM data for attitude estimation. The method has been evaluated using 1629 aerial images from an $80 \mathrm{~km}$ long flight at up to $600 \mathrm{~m}$ altitude in an area with woodlands, cities, lakes and the sea. Example images with the estimated horizon overlaid are shown in figure 1(b) and (c). The ground elevation in the flight area affecting the horizon ranges from sea level up to about $500 \mathrm{~m}$ altitude. Our vision-based method is primarily intended to support navigation for small UAVs that cannot carry highly accurate IMUs due to both cost and payload weight restrictions. 
The key components of the proposed method to achieve the high accuracy are:

1. The use of fisheye (omnidirectional) images, where a large portion of the horizon can be seen irrespectively of the vehicle orientation.

2. Accurate calibration of the fisheye lens, especially close to the fisheye border.

3. Accurate detection and extraction of the horizon line in the fisheye image.

4. Registration of the perceived horizon line in the image with the geometrical horizon from a DEM.

5. Taking into account the refraction of oblique rays in the earth atmosphere.

The use of these components in a horizon detection method for attitude estimation is motivated as follows. For an airborne vehicle, the use of omnidirectional images is appropriate to ensure that the horizon will be seen in the image even for large attitude angles. Furthermore, omnidirectional images contain information on a larger fraction of the complete horizon line compared to perspective images and this makes the method more robust to partial occlusion of the horizon. The requirement for accurate camera calibration is obvious but is hard to obtain close to the fisheye circle, i.e. almost perpendicular to the optical axis. For high accuracy attitude estimates, registration with a DEM is necessary unless the terrain is truly flat and level which is rarely the case. If a DEM is used, the true horizon profile must be extracted from the image for high accuracy attitude estimates. In our test flight, the true horizon was often offset 4-5 pixels from the ideal horizon ellipse on the image plane. Approximations with a straight line (perspective image) or a perfect ellipse (omnidirectional image) assuming a level terrain will generate attitude errors depending on the true ground topography.

Finally, refraction correction results in a further improvement of accuracy. For airborne vehicles at 500$1000 \mathrm{~m}$ altitude, the true incidence angle for refracted rays from the sea level horizon will be around $0.05^{\circ}$ $0.1^{\circ}$ greater than if assuming straight ray propagation. This deviation would generate errors and shows that for high accuracy attitude estimates, the refraction in the atmosphere needs to be taken into account.

The main contribution of this paper is a method to detect and extract the true horizon line in fisheye images. This is achieved by first estimating an approximate horizon line by edge detection and a probabilistic Hough voting scheme (Grelsson and Felsberg, 2013) assuming that the earth is an ideal sphere. The true horizon line in the image is then extracted from edge pixels in a thin band ranging from the approximate, ideal horizon to an outer limit in the image plane, depending on the ground elevation taken from the DEM.

A second contribution is the proposed method of using the extracted horizon line and the geometrically expected horizon for accurate calibration of the fisheye lens. This calibration method requires accurate knowledge of the $6 \mathrm{DOF}$ camera position when the images were captured. The proposed calibration method was employed to refine the camera calibration in our test flight.

A third contribution is taking the ray refraction in the atmosphere into account when computing the geometric horizon line from DEM data. If ray refraction is ignored, a systematic attitude estimate offset is introduced which is a significant error source for high accuracy attitude estimate methods.

This paper is organized as follows. Section 2 gives a review of image based methods for vehicle attitude estimation, focusing on methods using horizon detection, DEMs, or omnidirectional images. Section 3 presents the camera and earth models used. An overview of the attitude estimation method and its prerequisites is given in section 4. A detailed description of the proposed method is presented in section 5. In section 6, the flight trial performed for evaluation of the proposed method is described. Section 7 presents the results obtained with the attitude estimation method. It also provides an analysis to explain the results and the main error sources. Finally, the conclusions are given in section 8. 


\section{Related work}

Vision-based methods are frequently applied for attitude estimation of a vehicle. Our proposed method is designed for attitude estimation of an airborne vehicle and employ horizon detection in omnidirectional images with DEM based refinement. To the best of our knowledge no method exist that use all these components for attitude estimation and we focus on related methods which are based on horizon detection and contain at least one of the other components. The components employed in the related methods and the accuracy of the attiude estimates obtained are summarized in table 1.

Table 1: Related methods and results.

\begin{tabular}{|l|l|l|l|l|l|}
\hline First author & Vehicle & Camera & DEM & Accuracy pitch/roll & Comments \\
\hline Natraj & Airborne & Omnidir. & No & Mean error 2.5-3.0 & \\
Bazin & Airborne & Omnidir. & No & Not applicable & \\
Mondragon & Airborne & Omnidir. & No & RMSE 0.2-4.4 & Yaw angle estimated \\
Thurrowgood & Airborne & Omnidir. & No & Average error 1-2 & Manual horizon as ground truth \\
Moore 2011a & Airborne & Omnidir. & No & Average error 1.5 & Manual horizon as ground truth \\
Moore 2011b & Airborne & Omnidir. & No & Average error 1.5 & Yaw angle estimated \\
Grelsson & Airborne & Omnidir. & No & Not reported & No attitude ground truth available \\
Bao & Airborne & Perspective & No & Not reported & No attitude ground truth available \\
Dusha & Airborne & Perspective & No & $\sigma=1.8^{\circ}$ (flight 1) & Pose estimates filtered with EKF \\
& & & $\sigma=0.4-0.7^{\circ}($ flight 2) & \\
Dumble 2012 & Airborne & Perspective & No & $\sigma=0.9-1.0^{\circ}$ & \\
Dumble 2014 & Airborne & Perspective & Yes & $\sigma=0.07^{\circ}$ and $0.03^{\circ}$ & Location estimated, synthetic images \\
Gupta & Ground & Perspective & Yes & $2 \sigma=0.50^{\circ}$ and $0.26^{\circ}$ & IMU supported, yaw angle estimated \\
\hline
\end{tabular}

An overview of available methods where horizon detection is used to infer the camera attitude for airborne vehicles is given in (Shabayek et al., 2012). Work on horizon detection in omnidirectional images is rather limited. In (Natraj et al., 2013) horizon detection is used for attitude estimation in catadioptric images from a rural area. A sky/ground segmentation is performed based on pixel color content using Markov Random Fields. The detected horizon line is projected onto the unit sphere and a best fit to a plane on the sphere is computed, assuming a smooth horizon line and implicitly a level terrain. A method to extract the skyline in catadioptric IR images is proposed in (Bazin et al., 2009). It is also suggested in the paper that the extracted skyline could be registered with the skyline from a DEM for attitude estimation, but no registration method is proposed. The skyline is extracted in synthetic IR-like images since the catadioptric mirrors required for image formation are not commercially available.

For catadioptric images, a sky/ground segmentation method based on the pixel RGB color content in a pyramid segmentation is used in (Mondragon et al., 2010). Horizon pixels are projected onto the unit sphere to estimate the best fit of a plane which is then used to estimate the pitch and roll angles. The relative heading angle between images is estimated by searching for a best match using an image column shift, implicitly assuming a pure rotation around the vertical axis.

Spectral and intensity properties are used to classify sky and ground regions in the image in (Thurrowgood et al., 2010). The classification rule is precomputed and based on a training set of images. Horizon edges are fitted to a 3D plane on the viewsphere to infer the attitude angles. The same group of authors presents an online learning method to classify the image into sky/ground based on spectral information and brightness (Moore et al., 2011a). The aircraft attitude is obtained by exhaustively matching the classified input image against a database of ideal image classifications, representing all possible attitude combinations. The same authors add a visual compass to also estimate the heading angle in (Moore et al., 2011b). Stabilized panoramic views, compensated for the estimated pitch and rolled angles, are compared with a reference view to infer the change in heading direction.

For horizon detection and attitude estimation from aerial fisheye images, (Grelsson and Felsberg, 2013) use edge detection and a probabilistic Hough voting scheme. The method presented is used as a basis for the 
proposed attitude estimation method in this paper.

For perspective images there are two main approaches for horizon detection, either edge detection or sky/ground segmentation using pixel color characteristics. A common technique for edge based methods is presented in (Bao et al., 2003) where edge pixels vote for horizon line directions and positions in a Hough voting scheme to infer the camera pitch and roll angles. In (Dusha et al., 2011) a state vector consisting of the pitch and roll angles and the aircraft body rates are estimated from horizon detection and optical flow in images from a perspective, front-mounted camera. Edge detection and Hough voting is used to generate candidate horizon lines, and optical flow is used to reject lines that are not close to the true horizon.

In a recent work (Dumble and Gibbens, 2012) a search for horizon pixels is performed in each column based on gradient thresholding and pixel color content. They look for a contiguous line of horizon pixels extending across the whole image but do not require the line to be straight. A gradient threshold is varied until only one line across the image remains, which is taken as the horizon. The best fit of the contiguous line to a straight line is made, implying that valuable horizon profile information will not be used in the subsequent attitude estimation.

The same authors extend their work (Dumble and Gibbens, 2014) and now include alignment of the measured horizon profile with a reference horizon profile from a DEM to estimate the attitude as well as the location of the airborne platform. A set of four perspective cameras is used to obtain a complete $360^{\circ}$ view of the horizon. To speed up the method considerably, the horizon profile is pre-computed from DEM data at a set of 3D grid points. The horizon profile is extracted from the closest grid point to the assumed 3D position. Using a horizon profile transformation scheme, the reference horizon profile is transformed to the currently assumed 6 DoF position. To refine the estimated location and attitude of the platform, the total pitch angle difference between the measured horizon and the transformed reference horizon is iteratively minimized. To evaluate the method, synthetic images from a flight simulator program is used. Images are rendered from a virtual flight along a valley in the Alps where the horizon profile variance is very large. Presumably (not mentioned in the paper), the same flight simulator is used as a DEM to generate the reference horizon profiles. The results obtained are very impressive. The attitude errors are at least one order of magnitude smaller than for any previous method without DEM refinement. Since this method (Dumble and Gibbens, 2014) is the one most similar to ours, we will return to it in section 7.5 and present a more detailed comparison between this method and ours together with an analysis of the results obtained with the two methods.

There are several other methods proposed for attitude estimation using horizon silhouette matching in mountainous areas (Naval Jr. et al., 1997), (Behringer, 1998), (Woo et al., 2007), (Baatz et al., 2012). These methods often rely on detection of mountain peaks and we are looking for a more general method for horizon registration.

Gupta and Brennan present one approach for registering the true horizon line in an image with DEM data for refined attitude estimation for a ground vehicle (Gupta and Brennan, 2008). Information from a frontmounted perspective camera and an IMU is fused and registered with DEM data to estimate the absolute yaw, pitch and roll angles. The attitude accuracy obtained is better than for any method without DEM refinement but the accuracy is somewhat inferior to recent results (Dumble and Gibbens, 2014). The main reasons for this are probably the smaller FOV and a smaller horizon profile variance in the images.

We are not aware of any previous method for attitude estimation taking the ray refraction in the earth atmosphere into account. Equations for refracted ray propagation are given in (Gyer, 1996). The effect is small and the attitude errors induced are often smaller than the errors from the assumption that the horizon is generated by a level terrain. However, for high accuracy attitude measurements using the horizon from airborne vehicles, the ray refraction effect cannot be neglected. For aircraft flying at $500 \mathrm{~m}$ altitude, attitude errors in the order of $0.05^{\circ}$ are introduced if neglecting ray refraction to the ideal horizon at sea level. 


\section{Camera and earth models}

\subsection{Camera model}

The fisheye camera model used is illustrated in figure 2a). The fisheye lens is modelled as in (Active Vision Group, University of Oxford, 2013) by first projecting a world 3D point $\mathbf{X}$ onto the unit sphere as point $\mathbf{x}_{\mathbf{S}}$. The point on the unit sphere is then projected onto the normalized image plane $\pi_{\mathrm{u}}$ by a perspective camera model with its optical center displaced the distance $L$ from the center of the unit sphere. Note that the distance from the optical center to the normalized image plane is 1 (one), but it is not drawn to scale for clarity.

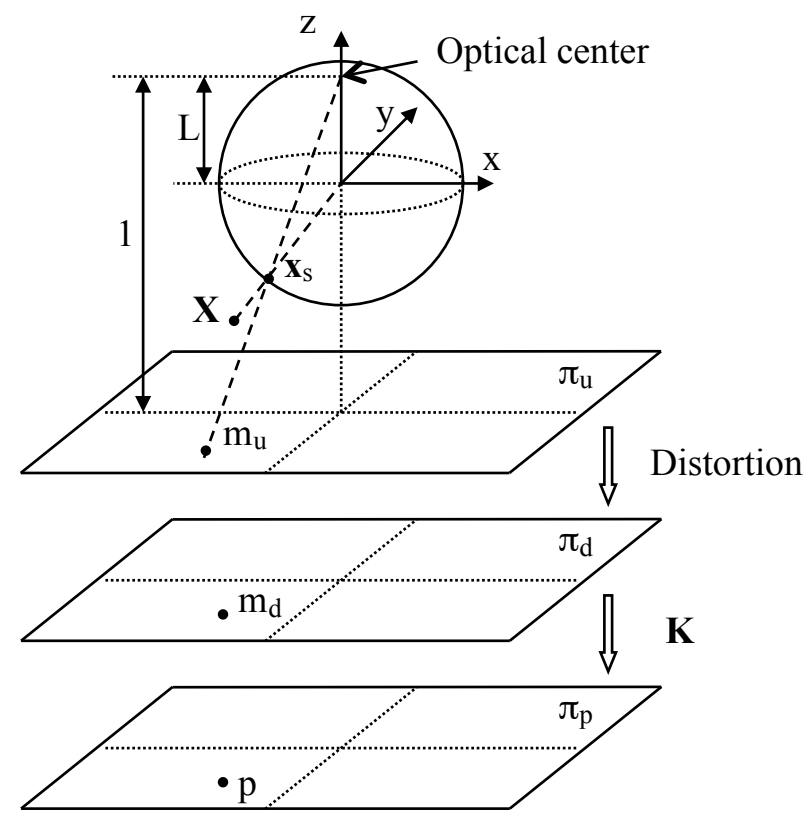

a)

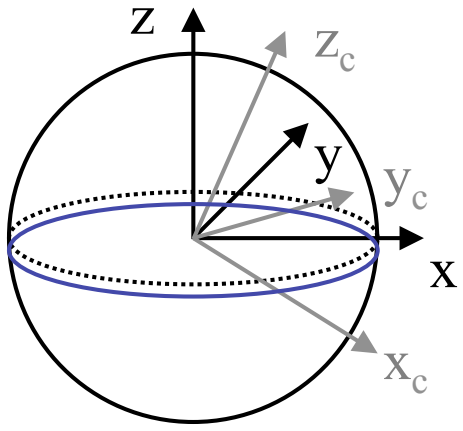

Figure 2: a) Fisheye camera model. b) Stabilized $(\mathrm{x}, \mathrm{y}, \mathrm{z})$ and rotated $\left(\mathrm{x}_{\mathrm{c}}, \mathrm{y}_{\mathrm{c}}, \mathrm{z}_{\mathrm{c}}\right)$ unit sphere.

Next, radial and tangential lens distortions are applied to adjust the undistorted point $m_{\mathrm{u}}=\left(u_{\mathrm{u}}, v_{\mathrm{u}}\right)$ to the distorted point $m_{\mathrm{d}}=\left(u_{\mathrm{d}}, v_{\mathrm{d}}\right)$. The model used for lens distortion is given in (1).

$$
\begin{aligned}
& r^{2}=u_{\mathrm{u}}^{2}+v_{\mathrm{u}}^{2} \\
& u_{\mathrm{d}}=u_{\mathrm{u}}\left(1+\sum_{i=1}^{4} k_{i} r^{2 i}\right)+2 t_{1} u_{\mathrm{u}} v_{\mathrm{u}}+t_{2}\left(r^{2}+2 u_{\mathrm{u}}^{2}\right) \\
& v_{\mathrm{d}}=v_{\mathrm{u}}\left(1+\sum_{i=1}^{4} k_{i} r^{2 i}\right)+2 t_{2} u_{\mathrm{u}} v_{\mathrm{u}}+t_{1}\left(r^{2}+2 v_{\mathrm{u}}^{2}\right)
\end{aligned}
$$

Compared to (Active Vision Group, University of Oxford, 2013), we have added radial distortion of degree 6 and 8 since this improved the accuracy of the camera model for pixels close to the fisheye circle. We denote the set of lens distortion parameters in (1) with $D$, containing four coefficients $k_{i}$ for radial distortion and two coefficients $t_{j}$ for tangential distortion. Finally, $m_{\mathrm{d}}$ is projected at point $p$ on the image plane $\pi_{\mathrm{p}}$ by applying the intrinsic camera parameter matrix $\mathbf{K}$.

For each image, the camera and hence the unit sphere rotates with the aircraft. All projections in the camera model description are made from the image plane onto the rotated unit sphere. For each image, 
we also consider a north-aligned and vertically stabilized unit sphere with the same center position as the rotated unit sphere. The ideal horizon from the sea level will generate a horizontal circle on the stabilized unit sphere, see figure $2 \mathrm{~b}$ ). The geometrical horizon from the DEM will be computed on the stabilized unit sphere. The camera rotation matrix ${ }^{b w} \mathbf{R}_{c}$ denotes the rotation from the stabilized unit sphere to the rotated unit sphere, i.e. it transforms a point ${ }^{\mathrm{w}} \mathbf{P}$ in the stabilized world frame to its coordinates ${ }^{\mathrm{b}} \mathbf{P}$ in the camera body frame such that

$$
{ }^{\mathrm{b}} \mathbf{P}={ }^{\mathrm{bw}} \mathbf{R}_{\mathrm{c}}{ }^{\mathrm{w}} \mathbf{P}
$$

\subsection{Earth model and refracted ray propagation}

We model the earth as a sphere with radius $R_{\mathrm{e}}=6371 \mathrm{~km}$. Overlaid on this sphere there is ground topography. For refracted ray propagation in the atmosphere around the earth, we use a spherically stratified model with thin radial layers of thickness $\Delta r$ and with constant refractive index $n$. The ray propagation model and its notation is taken from (Gyer, 1996). Using Snells law, it can be shown that a ray propagating through a spherically stratified model will follow a path as illustrated in figure 3 , which obeys the equation

$$
n r \sin \xi=n_{\mathrm{c}} r_{\mathrm{c}} \sin \xi_{\mathrm{c}}=n_{\mathrm{g}} r_{\mathrm{g}} \sin \xi_{\mathrm{g}}=k=\mathrm{constant}
$$

where $r=R_{\mathrm{e}}+h$ is the radius and $\xi$ is the incidence angle in the layer. The subscripts $c$ and $g$ denote the camera and the ground, respectively. The refractive index $n$ as a function of the altitude $h$ can be modelled, as a first order approximation, as

$$
n(h)=1+A \exp (-h / B) .
$$

In our work, we have used the constants $A=0.000292$ and $B=8400 \mathrm{~m}$, (National Physical Laboratory, 2013). This equation models the physical property employed in air pressure altimeters, that the air pressure drops exponentially with the altitude above the ground. Using equations (3) and (4), the incidence angle $\xi_{\mathrm{c}}$ for the horizon on a camera at altitude $h_{\mathrm{c}}$ can be determined, assuming that the perceived horizon is at sea level, $h=0$.

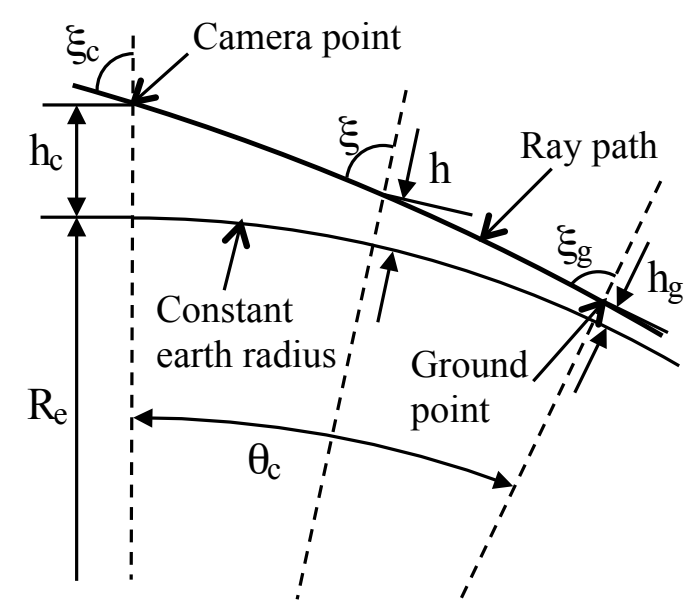

Figure 3: Refracted ray path.

\section{Attitude estimation method}

In this section, we present an overview of the main steps of the proposed attitude estimation method. We also list some system prerequisites for the method. Detailed descriptions of all steps in the attitude estimation method are given in section 5 . 


\subsection{Algorithmic steps, overview}

The algorithm can be described in six main steps, also shown in the flow chart in figure 4. Steps 1-3 are from (Grelsson and Felsberg, 2013), whereas the refinement in steps 4-6 is new. For each step in the overview, the section number is given where the detailed description can be found.

1. Run an edge detector on the input image. We have chosen the Canny detector (Canny, 1986) as it is robust and known to give no systematic offset in the edge location. (Section 5.1)

2. Estimation of the horizon normal vector on the unit sphere for each edge pixel. Project the edge pixel onto the unit sphere. The edge pixel direction on the image plane is projected as a tangent vector on the unit sphere. For a known vehicle altitude, the radius for the horizon on the unit sphere is deterministic. Combining this information geometrically, the horizon normal vector, i.e. the vehicle attitude, can be estimated for each edge pixel. (Section 5.2)

3. Probabilistic Hough voting (Hough, 1962) for all edge pixels to vote for the pitch and roll angles given an altitude estimate. The horizon on the unit sphere is derived from the estimated pitch and roll angles and the altitude. The voting allows the use of probability density functions for the vehicle altitude, and pitch and roll angles to make the voting more robust. (Section 5.3)

4. Extract edge pixels close the estimated horizon on the image plane and project the extracted horizon edge pixels onto the unit sphere. (Section 5.4)

5. Compute the geometrical horizon on the stabilized unit sphere from the DEM using an approximate vehicle position and heading as input. (Section 5.5)

6. The extracted horizon edge pixels from step 4 are registered with the geometrically computed horizon from step 5 to refine the initial vehicle attitude from step 3. (Section 5.6)

\subsection{System prerequisites}

There are four main system prerequisites for our proposed method to work.

1. The method requires a calibrated fisheye camera where the field of view (FOV) is larger than $180^{\circ}$ to ensure that part of the horizon is seen irrespective of the vehicle attitude. (Camera calibration, section 5.7)

2. A digital elevation model (DEM) of the flight area and its surroundings affecting the perceived horizon needs to be available. (DEM, section 5.8.1)

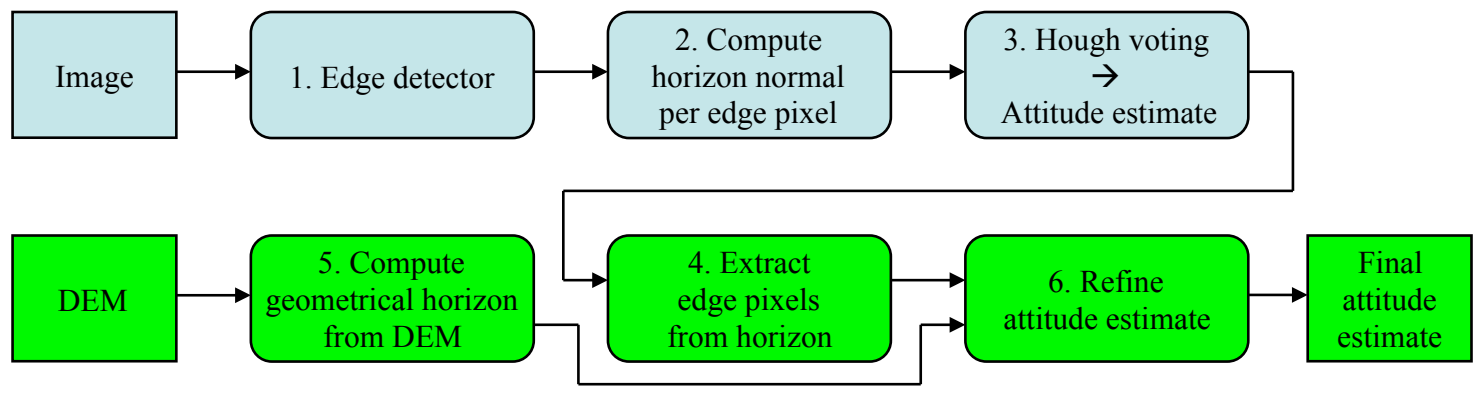

Figure 4: Algorithm flow chart. 
3. A function is required that computes the ray path in the earth atmosphere from an object at altitude $h_{\mathrm{obj}}$ to a camera at altitude $h_{\mathrm{c}}$ where the object and the camera are separated a distance $d$. To obtain an attitude estimation method with real-time capability, we have implemented this functionality as look-up tables as described in section 5.8.2.

4. An approximate vehicle position and heading is required to generate a geometric horizon from the DEM.

\section{Detailed algorithm description}

The first three subsections in the algorithm description, sections 5.1-5.3, are from (Grelsson and Felsberg, 2013), the remainder of the algorithm is new.

\subsection{Edge detector}

The first step in our method is an edge detection and we use the Canny detector (Canny, 1986) as it is known to give robust results with no systematic offset in the edge position. Before applying the Canny detector, the color image is converted to grayscale and the image is smoothed with a gaussian $3 \times 3$ kernel.

To reduce subsequent unnecessary computations in the Hough voting, we first remove some edge pixels that we know will not contribute to the horizon detection. In the edge image, there will be unwanted edge pixels due to the fisheye circle and the aircraft structure. These edge pixels will remain almost stationary on the image plane throughout a flight.

To determine a background edge map, we collected 100 edge images from a flight. Each pixel that was classified as an edge pixel in more than $30 \%$ of the images was considered to be a background edge pixel, i.e. originating either from the fisheye circle or from the aircraft structure. This background edge map is subtracted from the Canny edge map for each image prior to the Hough voting. The $30 \%$ threshold was set empirically and its exact value is not crucial for the attitude estimate results.

\subsection{Estimate horizon normal}

For an image edge pixel $p=(x, y)$, the projection onto the unit sphere is at point $P$. We compute the gradient in $p$ with $3 \times 3$ sobel filters, and define the edge direction in $p$ as $\left(-\nabla_{y}, \nabla_{x}\right)$, i.e. normal to the gradient. We define the image point $p_{\mathrm{e}}$ as the point one pixel away from $p$ along the edge direction. The projection of $p_{\mathrm{e}}$ onto the unit sphere is at $P_{\mathrm{e}}$. If $p$ is a horizon point, the vector $\overrightarrow{P P_{\mathrm{e}}}$ is a tangent vector on the unit sphere lying in the plane of the projected horizon. Let $\mathbf{t}$ be a vector of unit length in the direction of $\overrightarrow{P P_{\mathrm{e}}}$. If we look at a cross section of the unit sphere, orthogonal to the vector $\mathbf{t}$, as in figure 5 , we search for a second point $\mathrm{Q}$ in the plane of the horizon. For a certain altitude $h$, the radius of the horizon circle on the unit sphere is known. To find $\mathrm{Q}$, we define the vector

$$
\overrightarrow{O S}=\overrightarrow{O P} \times \mathbf{t}
$$

where $O$ is the origin in the unit sphere. We then obtain the vector

$$
\overrightarrow{O Q}=\overrightarrow{O P} \cos 2 \xi_{\mathrm{c}}+\overrightarrow{O S} \sin 2 \xi_{\mathrm{c}}
$$

where $\xi_{\mathrm{c}}$ is the incidence angle from the horizon for a camera at altitude $h_{\mathrm{c}}$. At this stage, we assume that the earth is smooth with radius $R_{\mathrm{e}}$. The points $Q_{\max }$ and $Q_{\min }$ denote the horizon points for the maximum and minimum altitudes given the probability distribution $p_{h}$ for the camera altitude in the subsequent voting. 


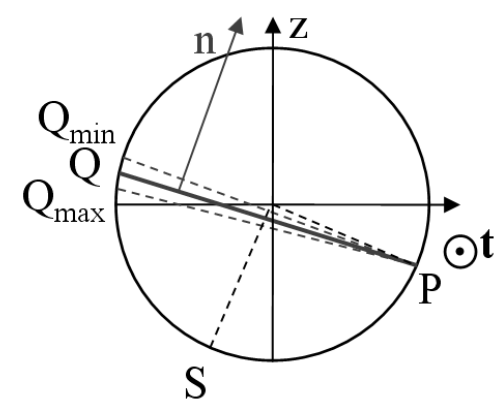

Figure 5: Estimate of horizon normal $\mathbf{n}$ from edge point. Tangent vector $\mathbf{t}$ is directed out of the paper.

A unit normal vector $\hat{\mathbf{n}}$ to the horizon plane can now be obtained as

$$
\hat{\mathbf{n}}=\frac{\overrightarrow{P Q} \times \mathbf{t}}{\|\overrightarrow{P Q} \times \mathbf{t}\|_{2}} .
$$

The pitch and roll angle estimates for the edge point $p$ are then given by

$$
\theta=\arcsin \hat{\mathbf{n}}_{y}, \quad \phi=-\arctan \frac{\hat{\mathbf{n}}_{x}}{\hat{\mathbf{n}}_{z}} .
$$

Note that angle estimates can easily be computed for various altitudes $h$. Vectors $\overrightarrow{O P}$ and $\overrightarrow{O S}$ remain constant, and it is only the angle $\xi_{\text {c }}$ that needs to be recomputed to get a new vector $\overrightarrow{O Q}$.

\subsection{Probabilistic Hough voting}

For each edge pixel $p$, we have shown how to compute the estimated pitch and roll angles for the horizon plane, given an assumed altitude $h$. It is then natural that the accumulator cells in our Hough voting is a pitch and roll angle grid. The cell resolution is adjusted to roughly correspond to the angular resolution of the aerial image. Minimum and maximum angles in the accumulator array are set to $\pm 80^{\circ}$ as this range covers the practical angles to be estimated for our flight. In the probabilistic Hough voting scheme, we want the weight $w$ for each vote to be proportional to the likelihood that the edge pixel is a horizon pixel given the probability distributions $p_{h}, p_{\theta}$ and $p_{\phi}$ for $h_{\mathrm{c}}, \theta$ and $\phi$, i.e. we want

$$
w(x, y) \propto \iiint p(x, y \mid h, \theta, \phi) d \phi d \theta d h
$$

Varying altitude. The true altitude above sea level can be measured either with a GPS or using an air pressure meter onboard the vehicle. In the computations, we have assumed that the estimated altitude is within $\pm 10 \%$ of the true altitude. For this relative altitude range, the variation in the estimated attitude is in the same order as the accumulator cell grid size. We have therefore chosen to divide the altitude range into rather few altitude segments, $N_{h}=11$, when calculating the weights. We set the weight $w_{h}$ for each segment to

$$
w_{h}=\int_{h_{\min }}^{h_{\max }} p_{h}(h) d h
$$

where $h_{\min }$ and $h_{\max }$ are the altitude limits for each segment. Note that these altitude weights can be precomputed for all edge pixels. 
Voting. Using Bayes' theorem and assuming that the probability distributions for $h_{\mathrm{c}}, \theta$ and $\phi$ are independent, we calculate the weights as

$$
w(x, y) \propto \int p_{h}(h) d h \int p_{\theta}(\theta) d \theta \int p_{\phi}(\phi) d \phi=w_{h} w_{\theta} w_{\phi}
$$

For each edge pixel $p$, we compute the estimated pitch and roll angles for each altitude $h$ and give a weighted vote in the nearest neighbor pitch-roll cell in the accumulator array. In our flight we had no prior information on the pitch and roll angles and the weights $w_{\theta}$ and $w_{\phi}$ were set to 1 . If you have prior information on the attitude angles, e.g. from an onboard IMU giving the relative rotation from the last absolute attitude estimate, the weights should be set in accordance with $p_{\theta}$ and $p_{\phi}$ over the pitch-roll grid.

Attitude estimate. In order to suppress local maxima, we convolve the values in the accumulator array with a gaussian kernel of size $7 \mathrm{x} 7$ and pick the index for the cell with maximum score as the attitude estimate. To refine the attitude estimate, we do a second order polynomial fit along the pitch and roll directions around the accumulator array maximum. The result after this step is an attitude estimate where we have assumed an ideal smooth earth with radius $R_{\mathrm{e}}$. The results obtained in this way have been analyzed in (Grelsson and Felsberg, 2013).

\subsection{Extract horizon edge pixels}

Using the method from (Grelsson and Felsberg, 2013), we noticed significant attitude estimate errors due to nonflat horizons and further refinement becomes necessary. To prepare for the refinement of the attitude estimate, we want to extract only the edge pixels originating from the horizon in the image. We do this geometrically and extract edge pixels close to the estimated horizon from the Hough voting. For a perfectly calibrated camera and exact knowledge of the camera altitude, the ellipse on the image plane corresponding to the estimated horizon from the Hough voting will always be slightly smaller than the true perceived horizon on the image due to the topography on top of the ideal spherical earth. Thus, most of the true horizon edge pixels will be on or outside the estimated horizon. Due to quantization effects in the edge detector (only integer pixels), some true horizon edge pixels may be $1 / 2$ pixel inside the estimated horizon. If the shift of the horizon due to topography is less than 1 pixel, it is sufficient to project the estimated horizon on the image plane and extract all edge pixels that are within a $3 \times 3$ matrix from the horizon pixels on the image plane.

For high resolution images, and when the ground elevation in the scene is large, the shift of the horizon due to topography may be larger than 1 pixel. In our flights, the shift from the ideal horizon was often in the order 4-5 pixels. To extract all true horizon edge pixels, we first compute the angular shift on the unit sphere generated by the highest elevation in the surrounding area compared to sea level. In our case, it was around $0.25^{\circ}$. We then add a $50 \%$ safety margin since the too small estimated ellipse will not be perfectly centered in the larger true horizon ellipse but often leans to one side. We set the upper angular limit to $0.4^{\circ}$ and denote it $\beta_{\text {lim. }}$. This means that all true horizon pixels on the image will be projected onto the rotated unit sphere in a thin band above the estimated horizon as given by the probabilistic Hough voting, see figure 6 . The black line $\mathbf{l}_{\mathrm{B}}$ in the figure is the estimated horizon. The green line $\mathbf{l}_{\mathrm{G}}$ is generated by points that make an angle $\beta_{\text {lim }}$ with the horizon points.

The line $\mathbf{l}_{\mathrm{B}}$, c.f. figure 6 , is defined by $(12)$. The line $\mathbf{l}_{\mathrm{s}}$ is the projection of the horizon onto the stabilized unit sphere, i.e. for a vertically oriented camera at altitude $h_{\mathrm{c}}$ where $\xi\left(h_{\mathrm{c}}\right)$ is the corresponding incidence angle on the camera. The line $\mathbf{l}_{\mathrm{s}}$ is then rotated with the estimated pitch and roll angles.

$$
\begin{aligned}
\mathbf{l}_{\mathrm{s}} & =\left\{\mathbf{x}_{\mathrm{s}} \mid x_{\mathrm{s}}^{2}+y_{\mathrm{s}}^{2}+z_{\mathrm{s}}^{2}=1 \wedge z_{\mathrm{s}}=\cos \left(\pi-\xi\left(h_{\mathrm{c}}\right)\right)\right\} \\
\mathbf{l}_{\mathrm{B}} & =\left\{\mathbf{x}_{\mathrm{B}} \mid \mathbf{x}_{\mathrm{B}}=\mathbf{R}\left(\theta_{\mathrm{est}}, \phi_{\mathrm{est}}\right) \mathbf{x}_{\mathrm{s}}, \mathbf{x}_{\mathrm{s}} \in \mathbf{l}_{\mathrm{s}}\right\}
\end{aligned}
$$




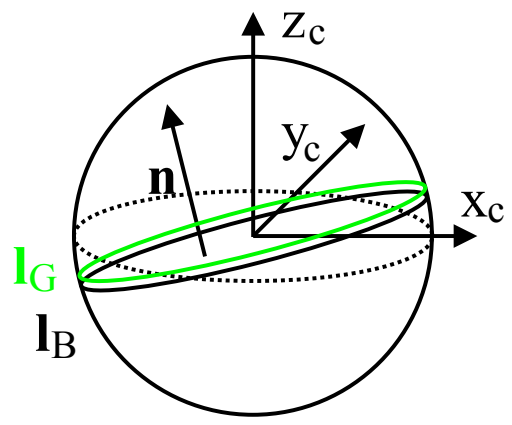

Figure 6: Band on rotated unit sphere with conceivable true horizon edge pixels.

The line $\mathbf{l}_{\mathrm{G}}$ is defined by (13). Compared to $\mathbf{l}_{\mathrm{s}}$, the line $\mathbf{l}_{\mathrm{sh}}$ is shifted an angle $\beta_{\text {lim }}$ upwards on the unit sphere.

$$
\begin{aligned}
\mathbf{l}_{\mathrm{sh}} & =\left\{\mathbf{x}_{\mathrm{sh}} \mid x_{\mathrm{sh}}^{2}+y_{\mathrm{sh}}^{2}+z_{\mathrm{sh}}^{2}=1 \wedge z_{\mathrm{sh}}=\cos \left(\pi-\xi\left(h_{\mathrm{c}}\right)-\beta_{\mathrm{lim}}\right)\right\} \\
\mathbf{l}_{\mathrm{G}} & =\left\{\mathbf{x}_{\mathrm{G}} \mid \mathbf{x}_{\mathrm{G}}=\mathbf{R}\left(\theta_{\mathrm{est}}, \phi_{\mathrm{est}}\right) \mathbf{x}_{\mathrm{sh}}, \mathbf{x}_{\mathrm{sh}} \in \mathbf{l}_{\mathrm{sh}}\right\}
\end{aligned}
$$

We project the band between the lines $\mathbf{l}_{\mathrm{B}}$ and $\mathbf{l}_{\mathrm{G}}$ onto the image plane to create a mask for potential horizon edge pixels. Since the edge detector gives integer pixel values, we include a $3 \times 3$ neighborhood around each projection point on the image plane in the mask. From the Canny edge image, we only extract the edge pixels within the mask for the subsequent attitude refining process.

\subsection{Compute geometrical horizon}

To compute the geometrical horizon, the availability of a DEM is required. In our case, we use the SRTM3 database, (NASA, 2013). See section 5.8.1 for more details. We also need an approximate global 3D position for the vehicle. This 3D position may be obtained from a GPS onboard the vehicle but it could equally well be estimated utilizing a ground terrain navigation method. These methods may be image based like the one proposed in (Grelsson et al., 2013).

For the maximum altitude in our flight, $600 \mathrm{~m}$, the distance to the ideal horizon is around $100 \mathrm{~km}$. To have some margin, we extract height profiles up to $200 \mathrm{~km}$ away from the vehicle. Based on the vehicle XY position, we extract the height profile in all angular directions $\alpha_{i}$ around the vehicle from the DEM, i.e. in all directions where we have extracted horizon edge pixels on the stabilized unit sphere. For each direction, we extract the elevation profile at every $100 \mathrm{~m}$, i.e. at about the same resolution as the DEM used. We use a bilinear interpolation to find the elevation between grid points in the SRTM3 database. An example elevation profile is shown in figure 7(a). The plateaus in the elevation profile are generated by the two large lakes Vättern and Vänern.

Next, we want to compute the largest incidence angle on the camera, located at altitude $h_{\mathrm{c}}$, that is generated by all land objects at altitudes and distances given by the extracted elevation profile. As it would be computationally too demanding to compute ray paths between all objects along the elevation profile and the camera online, we use look-up tables to find the incidence angle on the camera for all land objects along the elevation profile. The generation of LUTs is described in section 5.8.2. For the elevation profile in figure $7(\mathrm{a})$, the corresponding incidence angles on the camera are shown in figure $7(\mathrm{~b})$. We take the maximum of all incidence angles along the elevation profile, denoted $\xi_{\max }$, to be the geometrical horizon point for the angular direction $\alpha_{i}$ where the elevation profile is valid. This is repeated for all angular directions in the XY 

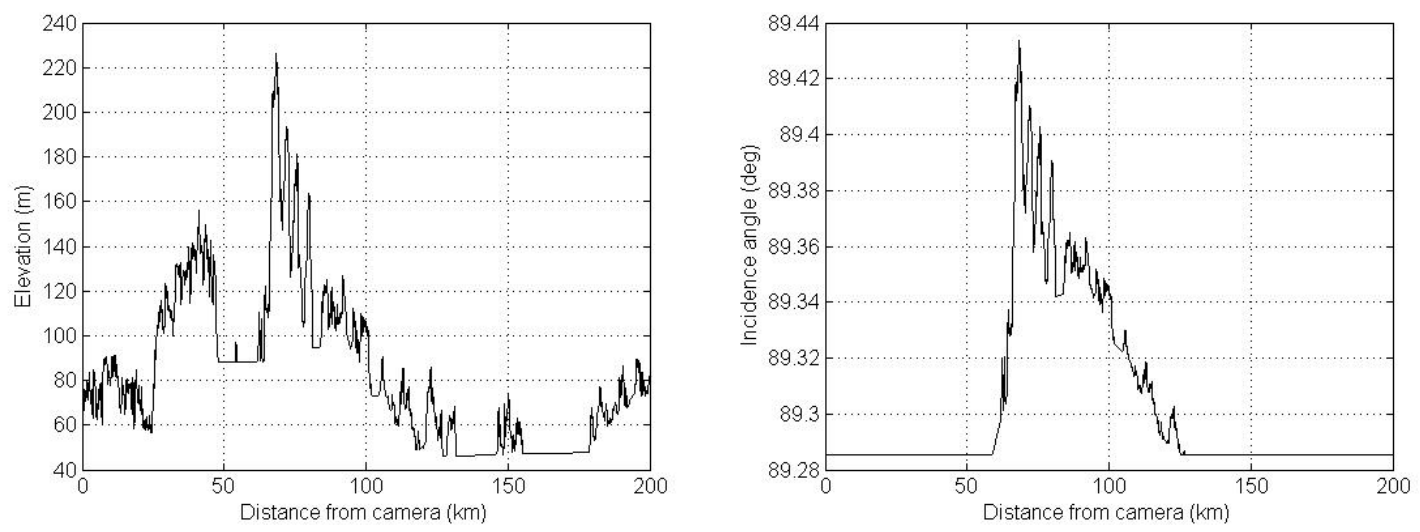

Figure 7: (a) Elevation profile and (b) corresponding incidence angle profile.

plane where we have horizon edge pixels on the stabilized unit sphere and results in a point set

$$
{ }^{\mathrm{w}} \mathbf{P}_{\text {geom }, i}=\left[\begin{array}{lll}
\cos \alpha_{i} & \sin \alpha_{i} & \cos \left(\pi-\xi_{\max , i}\right)
\end{array}\right]^{\mathrm{T}}
$$

for the geometrically computed horizon.

\subsection{Refine estimated attitude}

The method we use for refining the estimated vehicle attitude may also be used for calibration of the camera parameters and the camera mounting on the vehicle given that accurate position and attitudes are known from other sensors. The difference is only which parameters are kept constant in a nonlinear optimization process.

First, we denote the vehicle rotation matrix transforming points from a world coordinate frame to a vehicle body frame as ${ }^{b w} \mathbf{R}_{\text {veh }}$ and the corresponding camera rotation matrix as ${ }^{b w} \mathbf{R}_{\mathrm{c}}$. Assuming that the camera is not perfectly aligned with the aircraft body, there is a relative rotation ${ }^{\mathrm{cv}} \mathbf{R}_{\mathrm{rel}}$ between the vehicle frame and the camera frame defined as

$$
{ }^{\mathrm{c}} \mathbf{R}_{\mathrm{rel}}={ }^{\mathrm{bw}} \mathbf{R}_{\mathrm{c}}{ }^{\mathrm{bw}} \mathbf{R}_{\mathrm{veh}}^{\mathrm{T}}
$$

During the flight, the estimated vehicle orientation ${ }^{\mathrm{bw}} \mathbf{R}_{\mathrm{veh}, \mathrm{est}}$ is obtained from the estimated camera orientation ${ }^{\text {bw }} \mathbf{R}_{\mathrm{c}, \text { est }}$ as

$$
{ }^{\mathrm{bw}} \mathbf{R}_{\mathrm{veh}, \mathrm{est}}={ }^{\mathrm{cv}} \mathbf{R}_{\mathrm{rel}}^{\mathrm{T}}{ }^{\mathrm{bw}} \mathbf{R}_{\mathrm{c}, \mathrm{est}}
$$

Now, we use the extracted horizon edge pixels in the image (described in section 5.4). We backproject all horizon edge pixels onto the rotated unit sphere and denote the $3 \mathrm{D}$ point set on the sphere as ${ }^{\mathrm{b}} \mathbf{P}_{\mathrm{s}}$. Next, we rotate the point set with the transpose of the estimated camera rotation matrix given by the probabilistic Hough voting. The new rotated point set on the sphere

$$
{ }^{\mathrm{w}} \mathbf{P}_{\mathrm{r}}={ }^{\mathrm{bw}} \mathbf{R}_{\mathrm{c}, \text { est }}^{\mathrm{T}}{ }^{\mathrm{b}} \mathbf{P}_{\mathrm{s}}={ }^{\mathrm{bw}} \mathbf{R}_{\mathrm{veh}, \text { est }}^{\mathrm{T}}{ }^{\mathrm{c}} \mathbf{R}_{\mathrm{rel}}^{\mathrm{T}}{ }^{\mathrm{b}} \mathbf{P}_{\mathrm{s}}
$$

will ideally be the perceived horizon points rotated to the stabilized unit sphere, i.e. a camera system aligned with the world coordinate frame. With perfect image data, the rotated points ${ }^{w} \mathbf{P}_{\mathrm{r}}$ would correspond to the geometrically computed horizon points ${ }^{\mathrm{w}} \mathbf{P}_{\text {geom }}$. Note that we need at least an approximate vehicle heading angle estimate which could be obtained e.g. from feature point tracking in the image sequence.

The induced error function is to minimize the sum of the arc lengths on the unit sphere between the respective points in ${ }^{\mathrm{w}} \mathbf{P}_{\mathrm{r}}$ and ${ }^{\mathrm{w}} \mathbf{P}_{\text {geom }}$. If we choose to compute the point set ${ }^{\mathrm{w}} \mathbf{P}_{\text {geom }}$ for the angles

$$
\alpha_{i}=\tan ^{-1}\left(\mathrm{P}_{\mathrm{r}_{\mathrm{y}}, i} / \mathrm{P}_{\mathrm{r}_{\mathrm{x}}, i}\right)
$$


on the XY-plane, the small arcs between the point sets will have their main component along the z-axis of the unit sphere. Using a small arc angle approximation, we define the error function to be the squared distance between the z-coordinate for the point sets, i.e.

$$
\epsilon_{z}=\sum_{i}\left(\mathrm{P}_{\mathrm{r}_{\mathrm{z}}, i}-\mathrm{P}_{\text {geom }_{\mathrm{z}}, i}\right)^{2}=\sum_{i}\left(\mathrm{P}_{\mathrm{r}_{\mathrm{z}}, i}-\cos \left(\pi-\xi_{\max }\left(\alpha_{i}\right)\right)\right)^{2}
$$

where the summation is made over all extracted horizon edge points. For attitude refinement, we search for the camera orientation ${ }^{b w} \mathbf{R}_{\mathrm{c}}$ that minimizes the error function in (19), i.e.

$$
{ }^{\mathrm{bw}} \mathbf{R}_{\mathrm{c}, \mathrm{est}}=\underset{\mathrm{bw} \mathbf{R}_{\mathrm{c}}}{\operatorname{argmin}} \epsilon_{z}\left({ }^{\mathrm{bw}} \mathbf{R}_{\mathrm{c}}\right) .
$$

We minimize the error function using the Levenberg-Marquardt algorithm and initialize with the camera orientation matrix from the Hough voting.

\subsection{Camera calibration}

If there is, as in our case, a ground truth available for the 3D world position and orientation for some images, a similar scheme as for the attitude refinement may be used for calibration of the camera and its mounting on the airborne vehicle. However, a good initialization for the camera parameters and the camera rotation matrix is required. Since this camera calibration is an off-line process, we start by selecting about 10 images where, preferably, the extracted horizon edges are in good agreement with the visually expected horizon. Images are chosen where the horizon is located at different positions in the image, to improve the conditioning of the minimization problem.

One difference compared to the attitude refinement is that we now use the ground truth camera rotation matrix ${ }^{b w} \mathbf{R}_{c, \text { true }}$ to compute the rotated, backprojected points on the unit sphere.

$$
{ }^{\mathrm{w}} \mathbf{P}_{\mathrm{r}}={ }^{\mathrm{bw}} \mathbf{R}_{\mathrm{c}, \text { true }}^{\mathrm{T}} \mathbf{P}_{\mathrm{s}}={ }^{\mathrm{bw}} \mathbf{R}_{\mathrm{veh}, \text { true }}^{\mathrm{T}}{ }^{\mathrm{cv}} \mathbf{R}_{\mathrm{rel}}^{\mathrm{T}}{ }^{\mathrm{b}} \mathbf{P}_{\mathrm{s}}
$$

We minimize the same error function as in (19), but now sum over all horizon edge pixels in all of the chosen images, i.e.

$$
\left({ }^{\mathrm{bw}} \mathbf{R}_{\mathrm{rel}, \mathrm{est}}, \mathbf{K}_{\mathrm{est}}, \mathrm{D}_{\mathrm{est}}\right)=\underset{\text { bw } \mathbf{R}_{\mathrm{rel}}, \mathbf{K}, \mathrm{D}}{\operatorname{argmin}} \sum_{\text {im }} \epsilon_{z}\left(\mathrm{im},{ }^{\mathrm{bw}} \mathbf{R}_{\mathrm{rel}}, \mathbf{K}, \mathrm{D}\right) .
$$

Since we are now optimizing over 14 parameters in total, it is very likely that the Levenberg-Marquardt algorithm will end up in a local minimum for the error function. Therefore, this process has shown to improve the overall attitude estimation performance if a good initialization is provided.

\subsection{DEM and LUT}

\subsubsection{Digital Elevation model}

The DEM used in this paper is the freely available SRTM3 data from (NASA, 2013). The XY grid resolution is 3 arc-seconds or around $90 \mathrm{~m}$ at the equator. The standard deviation for the elevation error is claimed to be around $8 \mathrm{~m}$.

\subsubsection{Generation of LUT for topography}

For a spherically stratified model (Gyer, 1996) shows that the angle $\theta_{\mathrm{c}}$ from a ground point at altitude $h_{\mathrm{g}}$ to the camera at altitude $h_{\mathrm{c}}$ is given by

$$
\theta_{\mathrm{c}}=\int_{h_{\mathrm{g}}}^{h_{\mathrm{c}}} \frac{k}{r \sqrt{n^{2} r^{2}-k^{2}}} d r .
$$




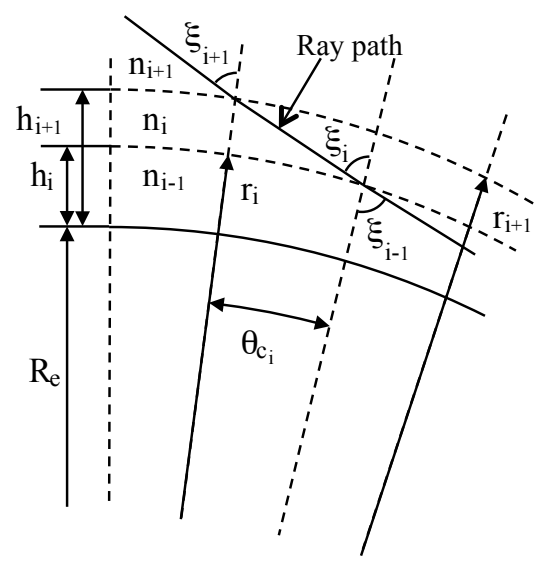

(a)
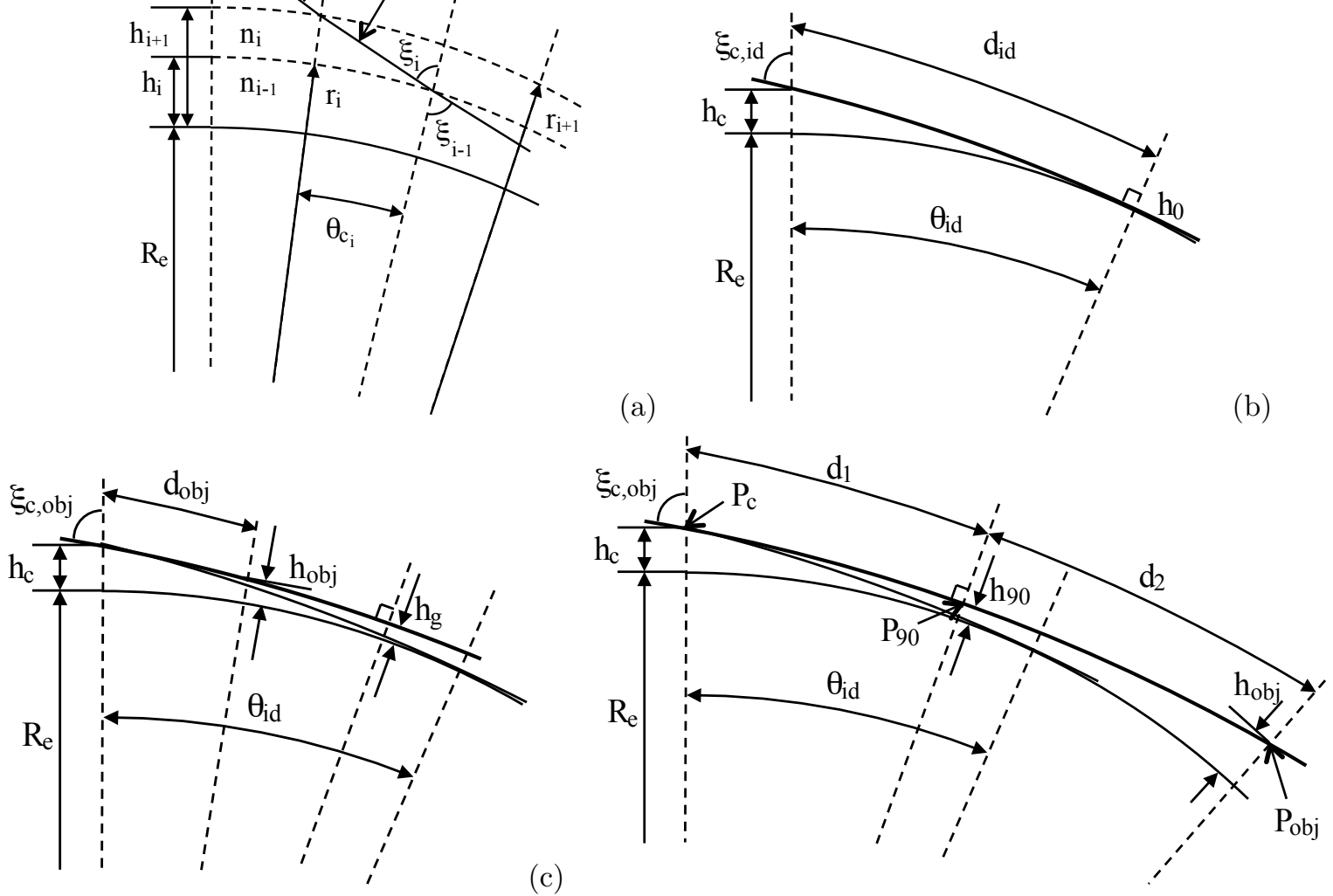

(d)

Figure 8: a) Refracted ray path in spherically stratified model. b) Refracted ray path with ideal horizon at sea level $h_{0}$. c) Refracted ray above ideal horizon passing an object at altitude $h_{\text {obj }}$ located the distance $d_{\text {obj }}$ from the camera. d) Same as c) but the object is located beyond the ideal horizon.

The parameter notation is from section 3.2 and the geometry is illustrated in figure 3. For a thin layer $i$ with constant refractive index $n_{i}$ in a spherically stratified model, see figure 8(a), the integral in (23) may be expressed as

$$
\theta_{c_{i}}=\int_{h_{i}}^{h_{i+1}} \frac{k / n_{i}}{r \sqrt{r^{2}-\left(k / n_{i}\right)^{2}}} d r=\cos ^{-1}\left(\frac{k}{n_{i} r_{i+1}}\right)-\cos ^{-1}\left(\frac{k}{n_{i} r_{i}}\right) .
$$

We further assume that the refractive index varies with the altitude as in (4). We set the camera height $h_{\mathrm{c}}$ constant, in our case somewhere in the range $200 \mathrm{~m}$ to $600 \mathrm{~m}$. First we assume that we view a spherical earth with no topography. The perceived horizon will then be at sea level, i.e. at altitude $h_{0}=0$, figure 8(b). At the horizon, the incidence angle is $\xi_{0}=\pi / 2$. We then use (24) to compute angular increments $\theta_{c_{i}}$ for each layer of thickness $d r$ up to the altitude $h_{\mathrm{c}}$. Adding these incremental steps will yield the distance to the ideal horizon

$$
d_{\mathrm{id}}\left(h_{\mathrm{c}}\right)=\sum_{h_{i}=0}^{h_{\mathrm{c}}} \theta_{\mathrm{c}_{i}} r_{i}
$$

From (3), the corresponding incidence angle on the camera $\xi_{\text {id }}\left(h_{\mathrm{c}}\right)$ can be computed. This determines the refracted ray path from the ideal horizon at sea level up to the camera at altitude $h_{\mathrm{c}}$.

If there would be no topography on a spherical earth with radius $R_{\mathrm{e}}$, this ray path would give the perceived horizon. The true perceived horizon (sky/ground boundary) may be shifted in the image for two reasons. 1) There is a land object at higher altitude than the ray path within the distance to the ideal horizon. 2) There 
is a land object furher away than the distance to the ideal horizon, but at sufficent height to be perceived at an incidence angle above the ideal horizon.

High objects within distance to ideal horizon. To compute this effect, consider a ground height $h_{\mathrm{g}}$ between 0 and $h_{\mathrm{c}}$. Set the incidence angle at the ground object $\xi_{\mathrm{g}}=\pi / 2$. Compute the ray path in the same manner as above until it reaches the altitude $h_{\mathrm{c}}$. Extract data points from the ray path at desired land object heights $h_{\mathrm{obj}}$ and record the corresponding distances to the camera $d_{\mathrm{c}, \mathrm{obj}}$ and the ray incidence angle on the camera $\xi_{\mathrm{obj}}$, see figure $8(\mathrm{c})$. Repeat the computations for all ground heights $h_{\mathrm{g}}$ from 0 to $h_{\mathrm{c}}$ in steps $\Delta h_{\mathrm{g}}$.

High objects beyond distance to ideal horizon. Even if an object is further away from the camera than the distance to the ideal horizon, the object may shift the sky/ground limit from the ideal case. The ray from the camera may take a path as in figure $8(\mathrm{~d})$ where the ray starts at point $P_{\mathrm{obj}}$, lowers in altitude from the ground object until it reaches the incidence angle $\pi / 2$ at point $P_{90}$ and then increases in altitude again until it reaches the camera at point $P_{c}$. To compute this effect, we start with a ray at altitude $h_{90}$ and set the incidence angle $\xi_{90}=\pi / 2$. We compute the ray path up to the maximum of the camera height $h_{c}$ and the highest object height $h_{\mathrm{obj}, \max }$ in the DEM. From this ray path, extract the distance to the camera $d_{1}$ from $P_{90}$ and the corresponding ray incidence angle on the camera $\xi_{\mathrm{c}, \mathrm{obj}}$. From the ray path on the right side of $P_{90}$, extract the distances $d_{\mathrm{r}}$ to the desired object heights $h_{\mathrm{obj}}$. Sum the distances $d_{1}$ and $d_{2}$ to obtain the total distance $d_{\mathrm{obj}}$ from the camera to the object at height $h_{\mathrm{obj}}$ and record together with the corresponding incidence angle $\xi_{\text {obj }}$. Repeat the computations for the altitude $h_{90}$ from 0 up to $h_{\mathrm{c}}$.

From the two cases 1 and 2, we can now extract for each camera height $h_{\mathrm{c}}$ and object height $h_{\mathrm{obj}}$, a point set with incidence angles and distances from the camera to the object. We resample this point set to obtain incidence angles at the desired step size for the distance, in our case at every $100 \mathrm{~m}$ up to $200 \mathrm{~km}$. We generated LUTs where the input parameters are camera height (increment $2 \mathrm{~m}$ ), object height (increment 2 $\mathrm{m}$ ), distance from camera (increment $100 \mathrm{~m}$ ) and the output parameter is the incidence angle on the camera.

We have only considered objects that are at a higher altitude than the camera if the objects are beyond the distance to the ideal horizon. The reason is that for the images considered in our test flight, there were no objects at a higher altitude than the camera within the distance to the ideal horizon.

\section{$6 \quad$ Flight trial}

To evaluate the proposed attitude estimation method, an aircraft flight trial with a fisheye lens camera was performed. A Nikon D800 with a Sigma f/8mm lens was mounted below the aircraft, figure 9. The image resolution is $6144 \mathrm{x} 4912$ pixels. The field-of-view is around $183^{\circ}$ resulting in a resolution around $0.04^{\circ} / \mathrm{px}$ for the full resolution images. For ease of camera mounting, the camera optical axis was not vertical but directed roughly $10^{\circ}$ to the right (starboard) and $10^{\circ}$ backwards (aft). This implies that when the aircraft was in level flight, the horizon was seen starboard (down in image) and aft (left in image) of the aircraft as shown in figure 10 .

The flight was carried out around Linköping in Sweden, see map in figure $11 \mathrm{a})-\mathrm{b})$, with ground elevations from sea surface up to around $500 \mathrm{~m}$ within a $200 \mathrm{~km}$ radius from the aircraft. Images were captured at around $1 \mathrm{fps}$ with flight altitudes up to $600 \mathrm{~m}$. The flight lasted for about 30 minutes and 1629 images were captured from $200 \mathrm{~m}$ altitude and above. These images are numbered 7795 to 9423 in the sequel. The flight altitude and the pitch and roll angles during flight are shown in figure $11 \mathrm{c})$-e).

Flight data was also recorded with a GPS and a highly accurate (tactical grade) IMU. The navigation sensor system used was a POS AV 510 from Applanix (Applanix, 2014). The ground truth 6DOF pose for the aircraft during the flight was obtained from an offline filtering process having navigation data from the complete flight available. A forward and backward filtering process was employed implying that the ground 

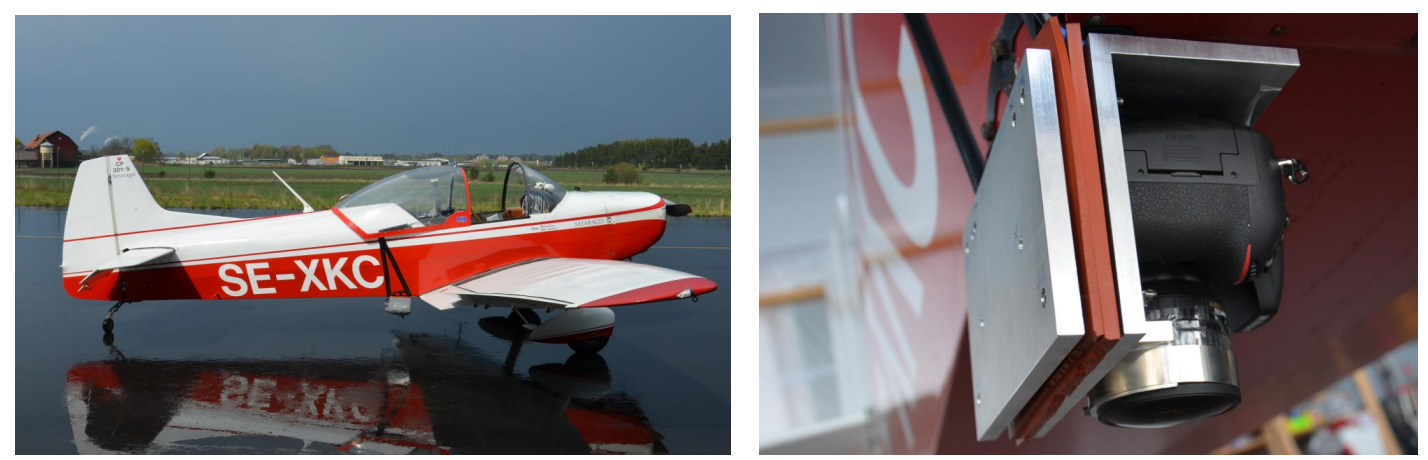

Figure 9: Aircraft camera mounting.

truth accuracy will not degrade with time during the flight (Groves, 2013). According to the manufacturer's specification for an RTX post-processed navigation solution, the ground truth position accuracy is claimed to be within one $\mathrm{dm}$ and the orientation accuracy within $0.005^{\circ}$ for the pitch and roll angles, and $0.008^{\circ}$ for the yaw angle, see table 2. The navigation sensors were mounted inside the cockpit and may not have experienced exactly the same accelerations and orientations as the camera mounted on the outside of the aircraft. The camera pose with respect to the aircraft was determined with level measurements, with an accuracy expected to be within $1.0^{\circ}$ for the respective Euler angles. A refined mounting pose was determined from the images as described in section 5.6.

The POS AV 510 system weighs around $4 \mathrm{~kg}$ and since it includes a highly accurate IMU it is relatively expensive. Cost and payload weight and size restrictions most often prevent such a navigation system to be employed on small UAVs.

Table 2: Extract of performance specification from Applanix. (Applanix, 2014)

\begin{tabular}{|l|c|c|c|}
\hline POS AV & 510 & 510 & 510 \\
& SPS & RTX & RTX Post-processed \\
\hline Position (m) & 1.5 Hor. & $<0.1$ Hor & $<0.1$ Hor. \\
& 3.0 Vert. & $<0.2$ Vert. & $<0.2$ Vert. \\
Roll and Pitch (deg) & 0.008 & 0.008 & 0.005 \\
True heading (deg) & 0.070 & 0.040 & 0.008 \\
\hline
\end{tabular}

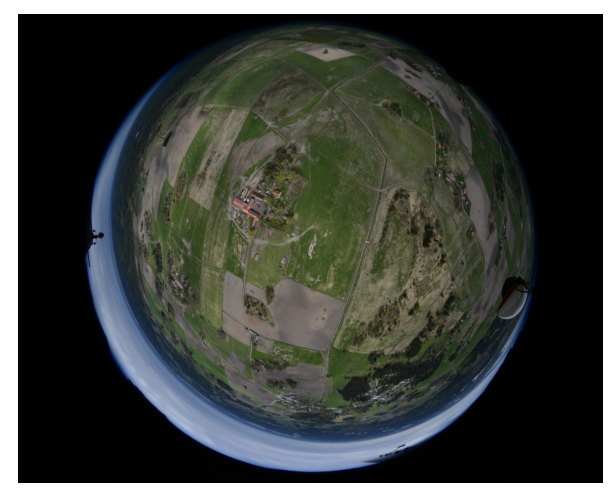

Figure 10: Typical image with aircraft in level flight. The horizon is seen starboard (down) and aft (left) of the aircraft. 


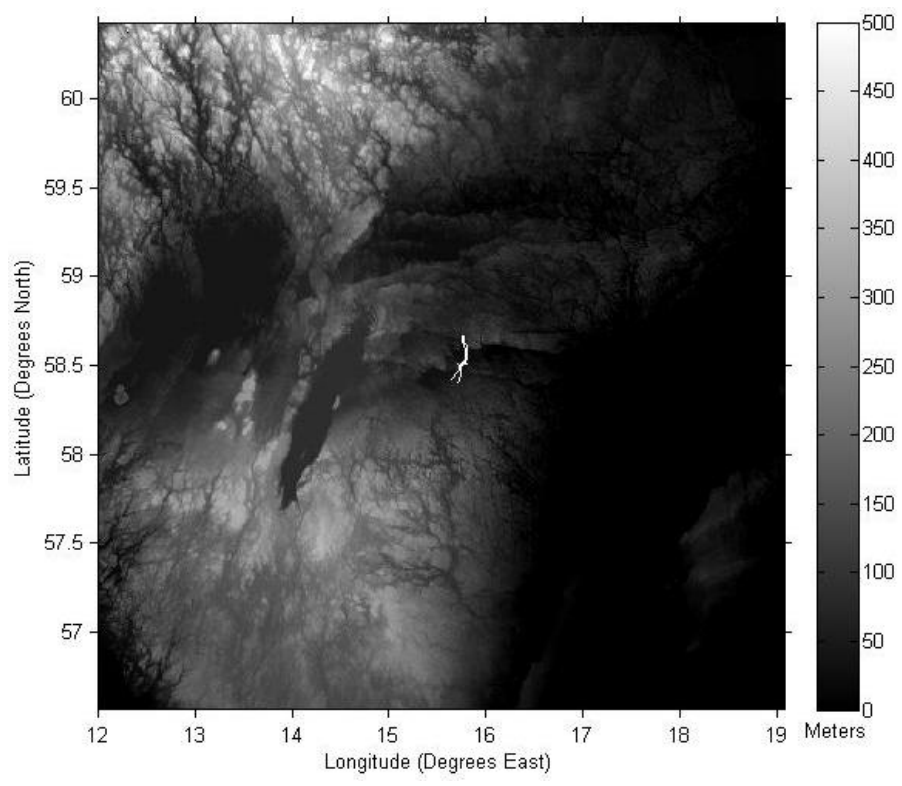

(a)

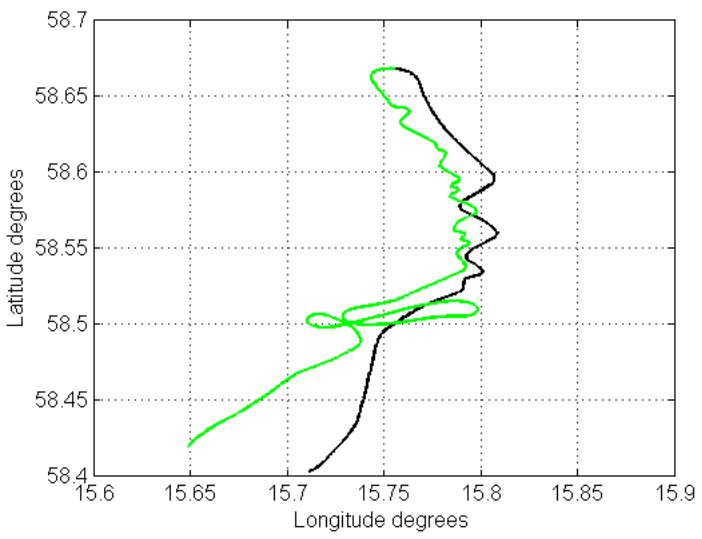

(b)

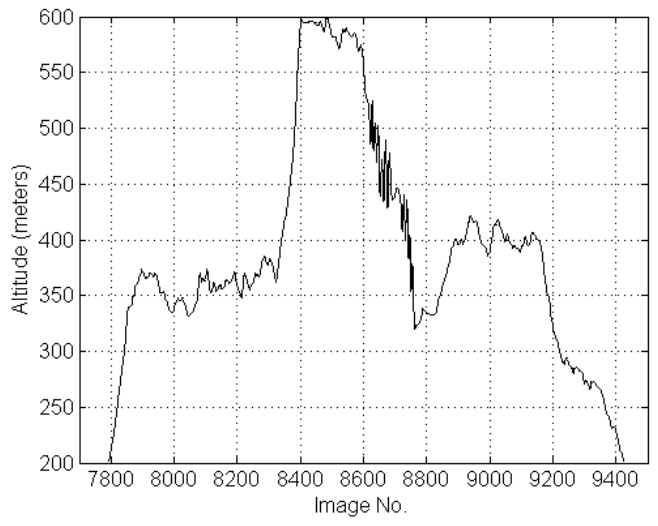

(c)
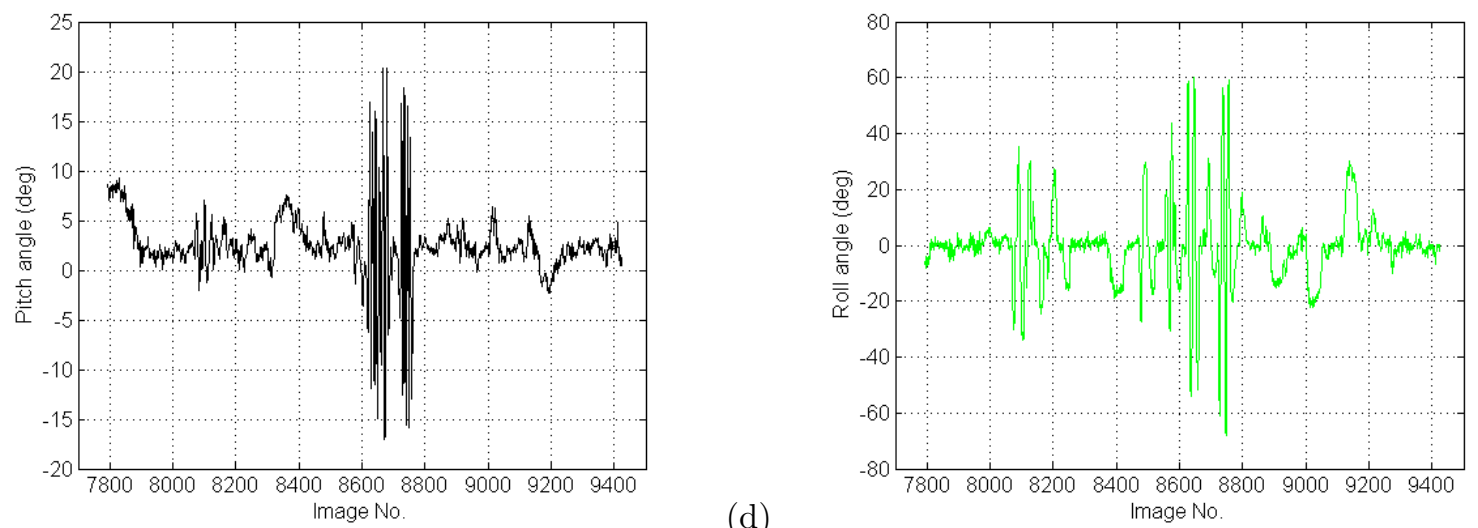

(d)

Figure 11: a) DEM with flight path overlaid (white) in central part of DEM. b) Zoom in of flight path. Northbound part (black), southbound part (green). c) Flight path altitude, d) pitch angle e) roll angle 


\section{Results and Discussion}

\subsection{Camera calibration}

Prior to evaluating the proposed attitude estimation method, the fisheye lens camera had to be calibrated. We first made a camera calibration using about 20 checkerboard images and the calibration tool provided by (Active Vision Group, University of Oxford, 2013). The checkerboard pattern comprised 8x5 $100 \mathrm{~mm}$ squares and was placed 3-5 meters from the camera. The calibration result was decent, but not accurate enough for our application, especially not for pixels close to the fisheye circle in the image. To improve the accuracy of the camera calibration, we used a set of 10 images from the flight where we extracted horizon edge pixels and matched these with the geometrically expected horizon from the DEM. The calibration method is described in section 5.6 and requires that the true camera position and orientation are known. In our case we used the ground truth from the navigation sensors as input for refining the camera calibration and the camera mounting rotation matrix. The checkerboard calibration results were used as an initialization for the camera calibration refinement.

\subsection{The Vättern effect}

Before we make a qualitative analysis of the attitude estimation results, we need to describe a geographical and geometrical effect on the image content that has a large impact on the results. As can be seen in figure 11(a), there is a long $(\sim 150 \mathrm{~km})$ and rather straight lake called Vättern west of the flight path. The width of the lake is $20-25 \mathrm{~km}$. When flying south, with the camera directed roughly $10^{\circ}$ starboard and aft, the lake generates a long edge in the image just inside the true horizon. The geometry is illustrated in figure 12 together with an example image and the corresponding edge map. Both the lake/land edge on the near side and the far side of the lake may show in the image depending on the image resolution. The far lake edge is at most $0.1-0.2^{\circ}$ from the true horizon and requires high image resolution to be resolved. The near lake edge is often around $0.5^{\circ}$ from the true horizon and may be seen for all image resolutions used.

In figure $12(\mathrm{~b})$ and (c), the near lake edge is readily resolved from the true horizon in the left part of the image. The two edges move closer and merge in the right part of the image where they cannot be resolved by the Canny edge detector. The edge in this part of the image is thus a mixture of the true horizon and the lake edge. The extent of this phenomenon, which we have called the Vättern effect, will of course be dependent on the geographical as well as the camera geometry for each individual image. Some examples of its impact on the attitude estimation results will be given later on.

Conceivably, there could be a reverse Vättern effect from clouds just above the horizon. We did not experience the clouds to be a problem in the images from our flight. One reason might be that the cloud edges are most often much shorter in length than the Vättern edges and hence will not significantly affect the Hough voting.

\subsection{Attitude estimation results}

To evaluate the proposed attitude estimation method, the camera attitude has been estimated for various image resolutions; the full resolution image and images downscaled a factor 2,4 and 8 . To determine the improvement obtained with the attitude DEM refinement process, a comparison is made for the results achieved with and without the refinement.

In order to make a fair comparison between results for different image scales, the camera calibration refinement for all scales should be made using the same set of images. For our flight trial this implied that we selected images from the first half of the flight for the calibration due to the Vättern effect. As stated above, the camera was mounted with its optical axis directed slightly to the right and backwards. The first half of 


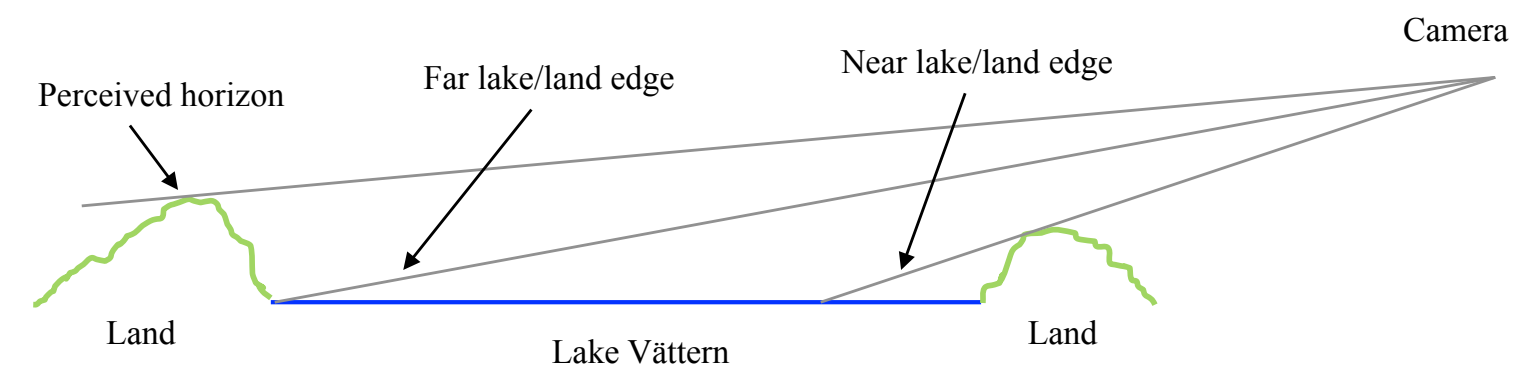

a)
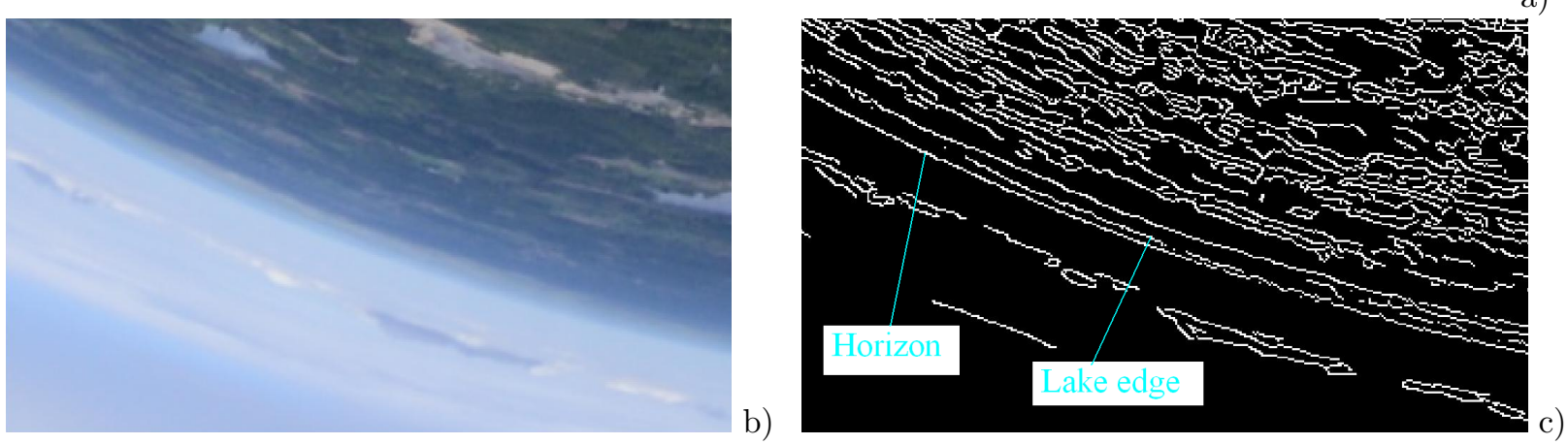

Figure 12: a) Geometry for the Vättern effect. b) Image 8420 downscaled a factor 2. c) The corresponding Canny edge map for b).

the flight (up to image 8400) was mainly northbound. In these images, the horizon will be seen facing east and south, unless making large manoeuvres, and there will be no lake edges. The return of the flight was mainly southbound, with the horizon facing west and north. For most of these images, the Vättern effect will be present to some extent. Consequently, only images from the northbound part of the flight were used for calibration refinement in the first part of the evaluation.

\subsubsection{Results without DEM refinement}

We first present results for attitude estimates after the Hough voting in the proposed method, i.e. no refinement based on the DEM has yet been performed. This stage corresponds to the method in (Grelsson and Felsberg, 2013). In figure 1, the estimated horizon is overlaid on two example images. From the images, it can be seen that there is a good fit between the estimated and true horizon, but nothing quantitatively can be said about the accuracy.

For all 1629 images, we computed the estimated pitch and roll angles and compared with the ground truth from the GPS/IMU sensors. The mean and standard deviation for the error in the pitch and roll angles over the 1629 images are listed in table 3. The total angular error in the table is defined as the square root of the sum of squared pitch and roll errors. The errors for all images are illustrated in figure 13. In general, the standard deviation for the pitch and roll angles decrease gently with image resolution. For the full resolution image and images downscaled a factor 2 and 4, there is a small positive mean error for both the pitch and roll angles whereas the mean error is considerably smaller for images downscaled a factor 8 . The mean and standard deviation for the total error agrees well with what can be expected for a Rician distribution given the errors for the pitch and roll angles.

To explain the results we start by illustrating how the horizon moves in the image when changing the aircraft pitch and roll angles. When in relatively level flight, the horizon will move left in the image with increasing pitch angle and down in the image with increasing roll angle, as shown in figure 14 a) and b). Without DEM refinement, we are looking for the horizon in the image as obtained from a smooth earth without topography. Due to topography, the true horizon in the image will lie slightly outside the ideal horizon. This slight shift 
Downscaled a factor 8
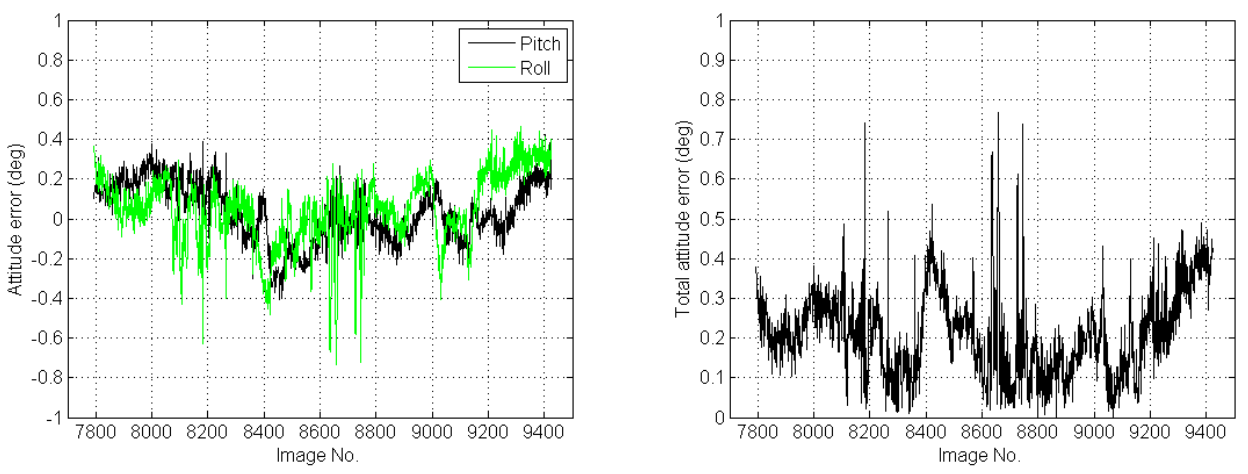

Downscaled a factor 4
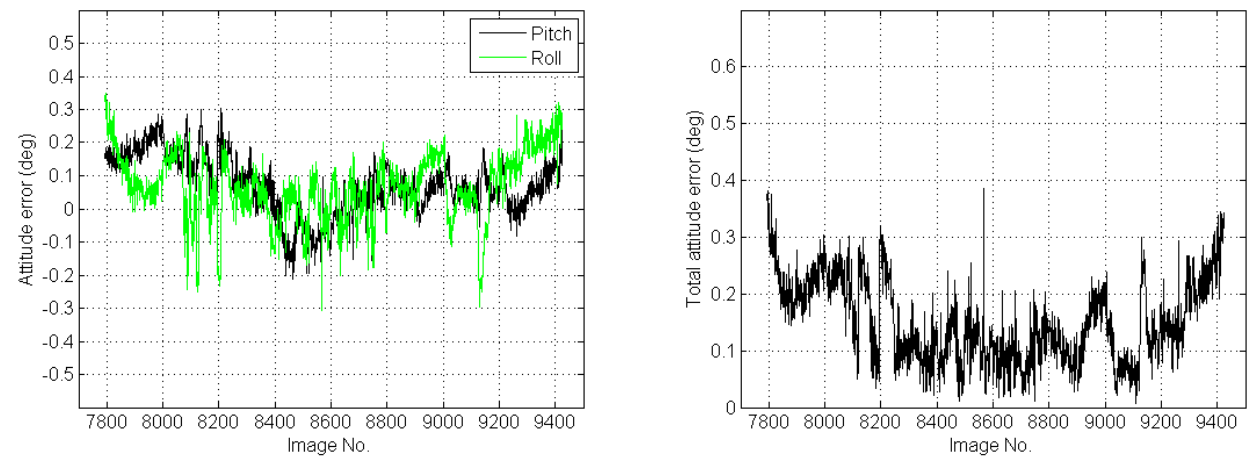

Downscaled a factor 2
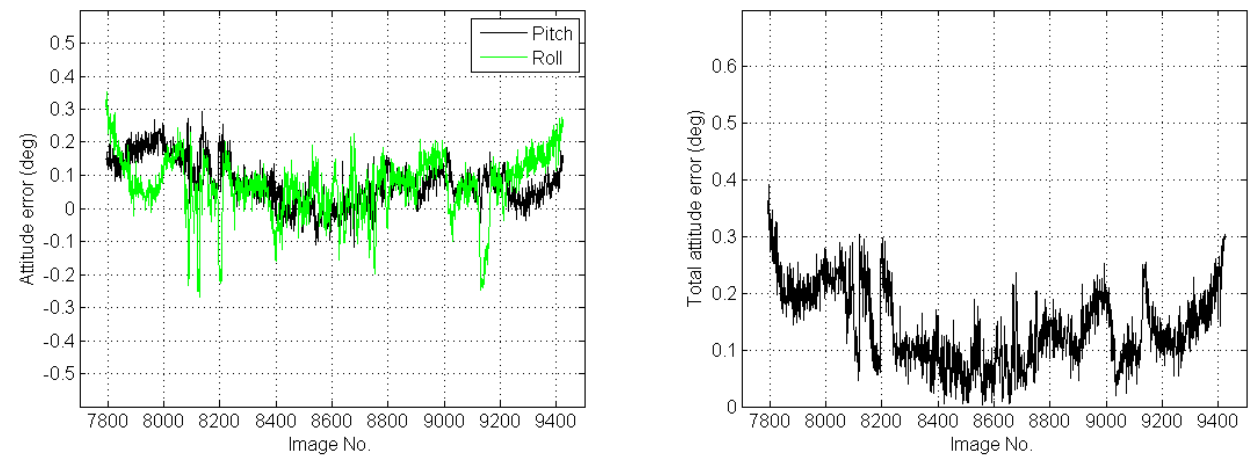

Full resolution
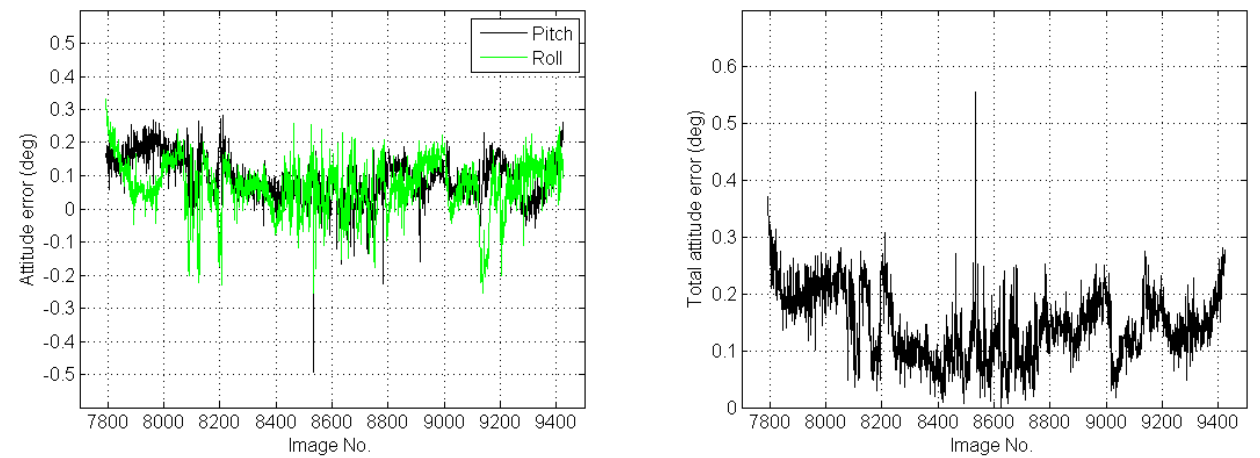

Figure 13: Results without refinement. Images downscaled a factor 8, 4, 2 and full resolution images. Note the different axis for the top figures. 
Table 3: Attitude estimation errors in degrees without DEM refinement.

\begin{tabular}{|c|c|c|c|c|c|c|c|}
\hline $\begin{array}{c}\text { Scale } \\
\text { factor }\end{array}$ & $\begin{array}{c}\text { Image } \\
\text { size }\end{array}$ & $\begin{array}{c}\text { Pitch } \\
\text { mean }\end{array}$ & $\begin{array}{c}\text { Pitch } \\
\text { std }\end{array}$ & $\begin{array}{c}\text { Roll } \\
\text { mean }\end{array}$ & $\begin{array}{c}\text { Roll } \\
\text { std }\end{array}$ & $\begin{array}{c}\text { Total } \\
\text { mean }\end{array}$ & $\begin{array}{c}\text { Total } \\
\text { std }\end{array}$ \\
\hline 8 & $0.8 \mathrm{Kx} 0.6 \mathrm{~K}$ & 0.033 & 0.148 & 0.044 & 0.181 & 0.213 & 0.111 \\
4 & $1.5 \mathrm{~K} \times 1.2 \mathrm{~K}$ & 0.071 & 0.091 & 0.064 & 0.103 & 0.150 & 0.073 \\
2 & $3 \mathrm{~K} \times 2.5 \mathrm{~K}$ & 0.081 & 0.068 & 0.068 & 0.092 & 0.140 & 0.068 \\
Full & $6 \mathrm{~K} \times 5 \mathrm{~K}$ & 0.092 & 0.068 & 0.067 & 0.085 & 0.145 & 0.063 \\
\hline
\end{tabular}

of the horizon to the left and down in the image compared to the ideal horizon will generate a small positive error on both the pitch and the roll angles. This explains the small positive mean error for the pitch and roll angles for the higher image resolutions. We stated earlier that the maximum angular shift of the horizon caused by the topography in the flight area was around $0.25^{\circ}$. The mean errors and standard deviations for the pitch and roll angles are about half this angle, i.e. in the correct order of magnitude.

For large positive aircraft roll angles (larger than the camera mounting angle) and small pitch angles, the horizon will move right in the image with decreasing pitch angle and down in the image with increasing roll angle, as shown in figure $14 \mathrm{c}$ ) and d). Due to ground topography, the true horizon will still be slightly outside the ideal horizon. For these images, the shift of the horizon to the left and up in the image will generate a small positive pitch error and a small negative roll error. This explains the negative roll errors obtained for images numbered around 8090, 8125, 8205 and 9140 where the aircraft roll angles are large and the pitch angles are small. Analogous explanations can be given for the attitude errors obtained for the large aircraft manoeuvres between images 8600 and 8800 .

For the lowest image resolution (downscaled a factor 8) there is a band for images 8400 to 8600 , corresponding to higher flight altitudes, where the attitude errors are generally negative. At higher altitudes, a large part of the horizon could not be resolved from the stronger lake edge in the Canny detector. The lake edge lies inside the ideal horizon and will thus generate negative pitch and roll errors. For higher image resolutions (full resolution and downscaled a factor 2), the ability to resolve the horizon from the lake improves and the trend with negative errors for these images diminishes.

For a few single images, there are quite large errors; image 9025 downscaled a factor 8 and images 8481 and 8533 in full resolution. For these images, the lake edge together with part of the horizon generate a higher peak in the Hough voting than the true peak from the horizon, thus creating an erroneous attitude estimate.

\subsubsection{Results after DEM refinement}

The attitude estimate results obtained after DEM refinement are listed in table 4 and illustrated in figure 15. These results are achieved with the same camera calibration as without DEM refinement.

Table 4: Attitude estimation errors in degrees after DEM refinement. Calibration with images from northbound part of flight.

\begin{tabular}{|c|c|c|c|c|c|c|c|}
\hline $\begin{array}{c}\text { Scale } \\
\text { factor }\end{array}$ & $\begin{array}{c}\text { Image } \\
\text { size }\end{array}$ & $\begin{array}{c}\text { Pitch } \\
\text { mean }\end{array}$ & $\begin{array}{c}\text { Pitch } \\
\text { std }\end{array}$ & $\begin{array}{c}\text { Roll } \\
\text { mean }\end{array}$ & $\begin{array}{c}\text { Roll } \\
\text { std }\end{array}$ & $\begin{array}{c}\text { Total } \\
\text { mean }\end{array}$ & $\begin{array}{c}\text { Total } \\
\text { std }\end{array}$ \\
\hline 8 & $0.8 \mathrm{~K} \times 0.6 \mathrm{~K}$ & -0.068 & 0.128 & -0.032 & 0.148 & 0.165 & 0.128 \\
4 & $1.5 \mathrm{~K} \times 1.2 \mathrm{~K}$ & -0.009 & 0.061 & -0.029 & 0.072 & 0.085 & 0.050 \\
2 & $3 \mathrm{~K} \times 2.5 \mathrm{~K}$ & 0.008 & 0.039 & -0.022 & 0.053 & 0.061 & 0.033 \\
Full & $6 \mathrm{~K} 5 \mathrm{~K}$ & 0.017 & 0.036 & -0.019 & 0.049 & 0.055 & 0.036 \\
\hline
\end{tabular}

The general trend is that the accuracy of the attitude estimates improves with image resolution. There is also a significant improvement compared to the results obtained without DEM refinement. Another observation is that the results for all scales are more accurate for the northbound part of the flight, i.e. up to image 8400 . For these images. the errors are smaller than the values listed in table 4 . The only spikes with errors up to 


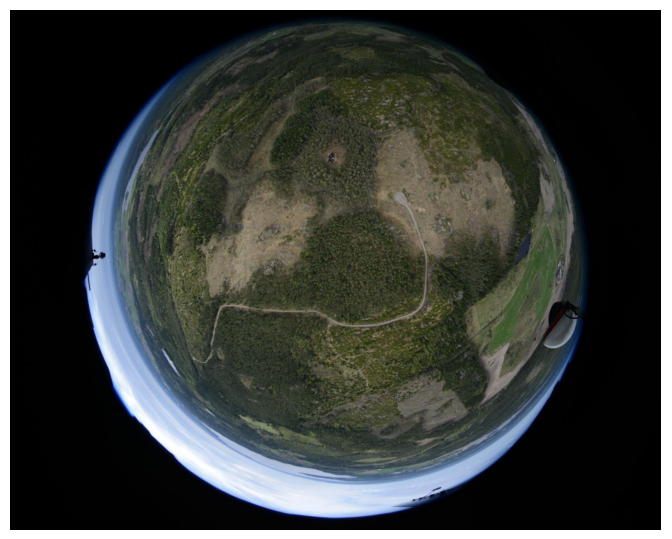

a) pitch $=0.7^{\circ}$, roll $=-0.9^{\circ}$

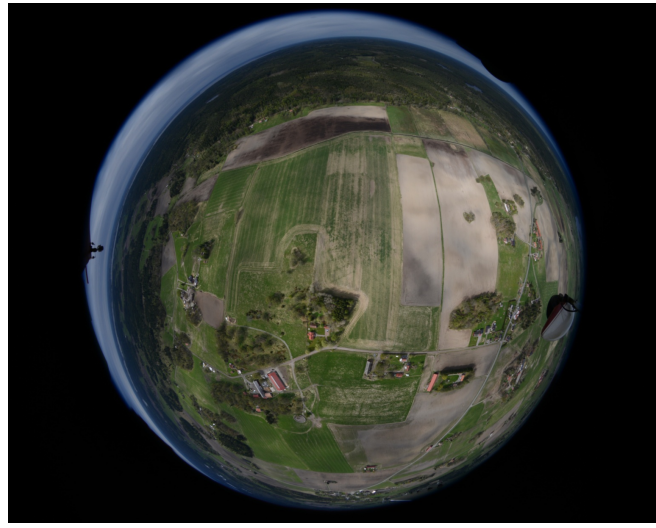

c) pitch $=3.1^{\circ}$, roll $=21.4^{\circ}$

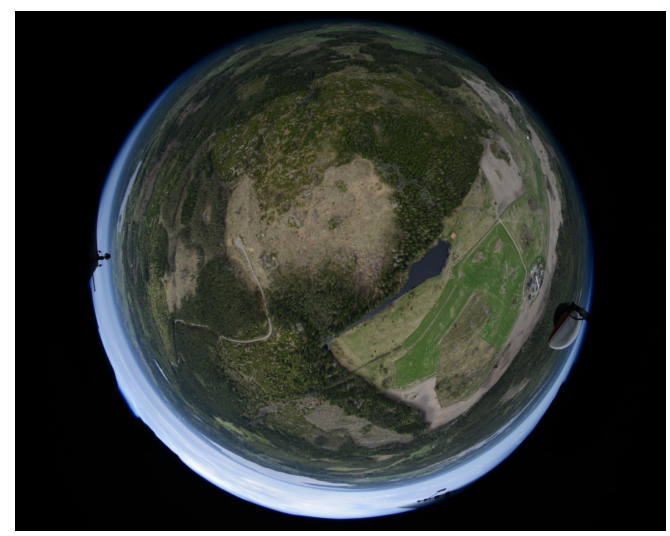

b) pitch $=4.8^{\circ}$, roll $=1.9^{\circ}$

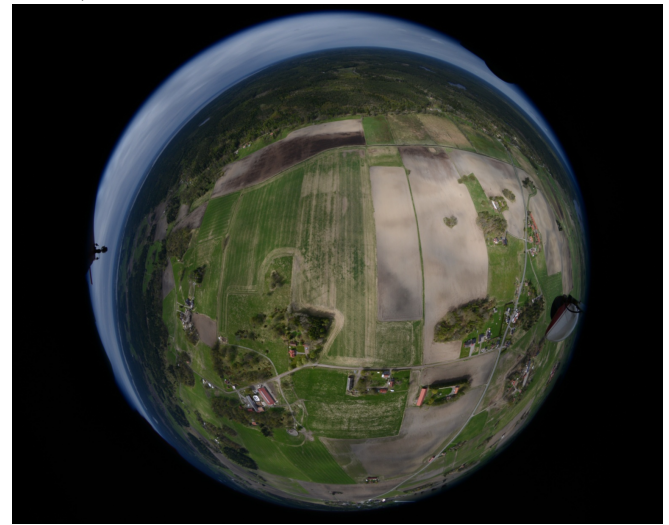

d) pitch $=2.4^{\circ}$, roll $=25.7^{\circ}$

Figure 14: Horizon movement upon changes in aircraft pitch and roll angles. The horizon moves left and down from a) to b). The horizon moves right and down from c) to d).

$0.1^{\circ}$ for these images occur for large aircraft roll angles. An example image from the northbound part of the flight (image 8280 downscaled a factor 2) and the corresponding edge map are shown in figure 16 a) and b). The edge pixels are colored cyan and the estimated horizon pixels after the Hough voting (without DEM refinement) are colored red. There are no other edge pixels close to the estimated horizon $\left(\right.$ within $\left.\sim 0.5^{\circ}\right)$. This means that the extracted horizon edge pixels from the total image will include almost no erroneous edge pixels and provide for an accurate attitude estimate through DEM refinement. The attitude estimate errors for this image and scale were less than $0.03^{\circ}$ which is smaller than the pixel size.

For the southbound part of the flight, after image 8400, the mean error for both attitude angles is negative and the standard deviation is larger than for the northbound part of the flight. For lower image resolutions (downscaled a factor 4 and 8) there are quite large parts in the image, especially for images 8400 to 8600 , where the horizon could not be resolved from the stronger lake edge in the Canny detector. When the perceived horizon edge on the image falls inside the geometrically expected horizon from the DEM, this generates negative errors on the pitch and roll angles.

For high image resolutions (full resolution and downscaled a factor 2), there are other modes inducing errors. An example is shown for image 8420 downscaled a factor 2 in figure $16 \mathrm{c}$ ) and d). The lake edge is either very close to the true horizon or merges with the horizon in the edge map. This will pull the estimated horizon inside the true horizon. Hence, the extracted horizon edge pixels will include, apart from the true horizon, both pure lake edges and edge pixels which are a mixture of the horizon and lake edges. The extracted horizon edge pixels inside the geometrically expected horizon will generate negative errors on the pitch and roll angle estimates, c.f. figure 14 a) and b). The errors for image 8420 downscaled a factor 2 was $-0.04^{\circ}$ and 
Downscaled a factor 8
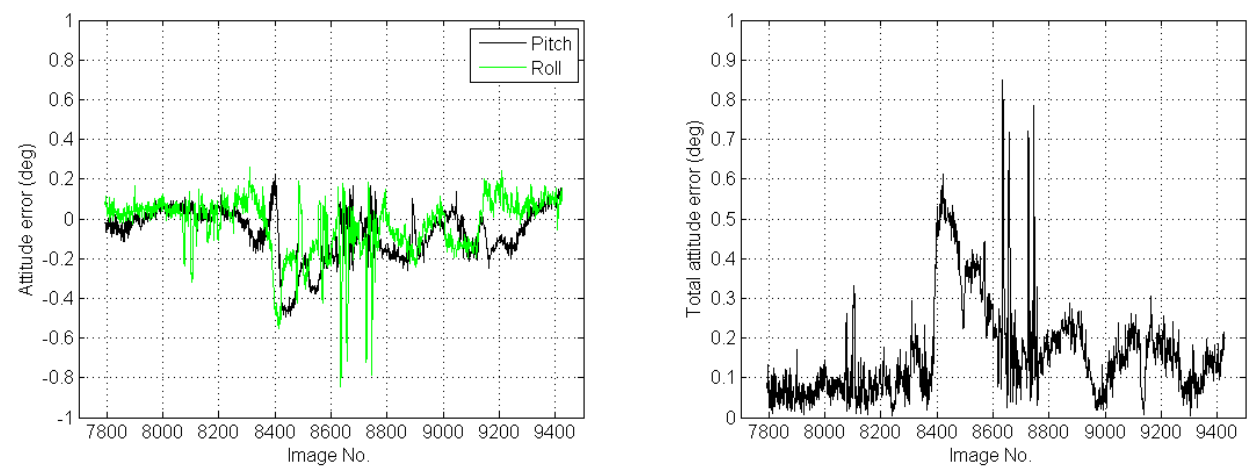

Downscaled a factor 4
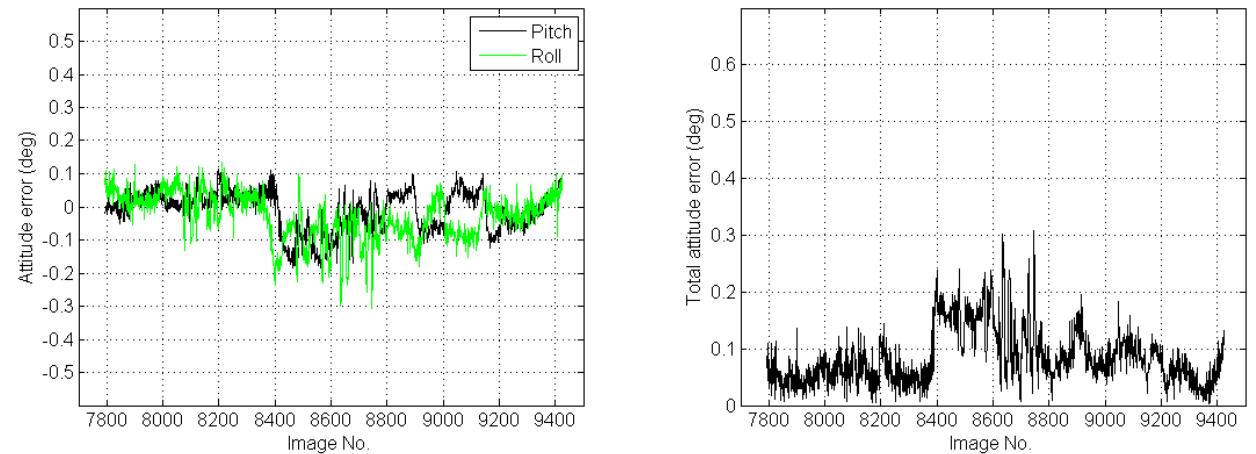

Downscaled a factor 2
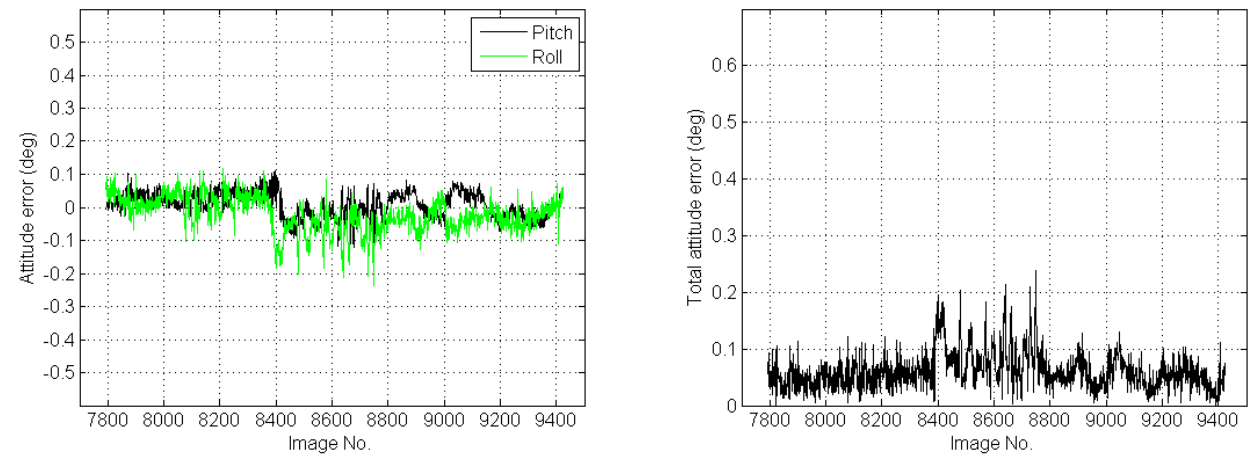

Full resolution
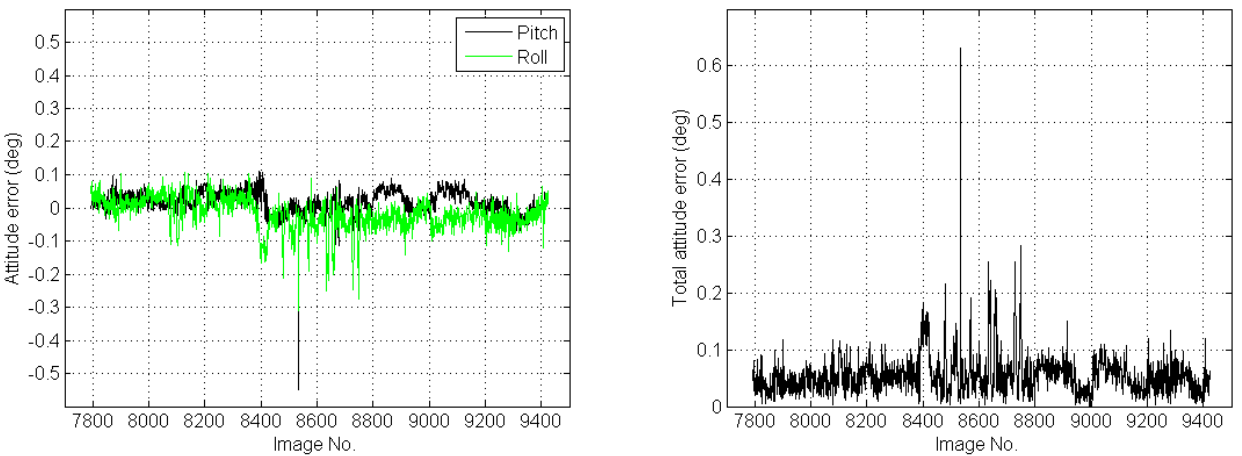

Figure 15: Results after DEM refinement. Calibration with images from northbound part of flight. Images downscaled a factor 8, 4, 2 and full resolution images. Note the different axis for the top figures. 

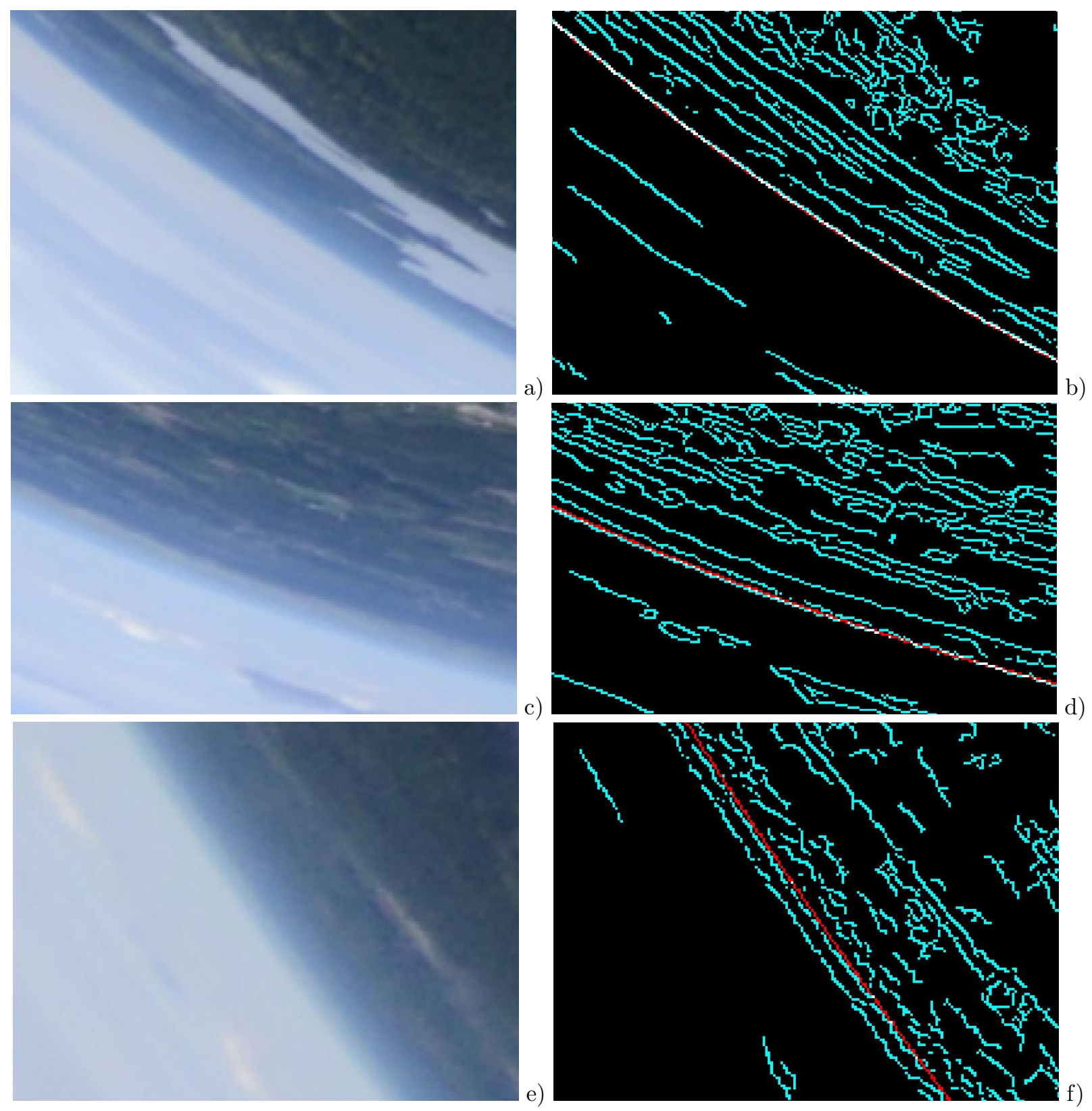

d)

Figure 16: Images and Canny edge maps (cyan) with estimated horizon after Hough voting overlaid (red). Image 8280 downscaled a factor 2 a),b), image 8420 downscaled a factor 2 c),d), image 8913 in full resolution e),f). 
$-0.15^{\circ}$ for the pitch and roll angles respectively.

Another example is image 8913 in full resolution shown in figure 16 e) and $\mathrm{f}$ ). Here, the lake edge and the true horizon can be seen as almost parallel edges separated around $0.15^{\circ}$. The estimated horizon assuming an ideal earth, which has a smaller radius than the true horizon, almost coincides with the lake edge in this zoomed part of the total image. Hence, when extracting horizon edge pixels, both the lake edges and the true horizon edges will be included. The lake edges lie inside the geometrically expected horizon and will induce negative errors on the pitch and roll angle estimates. In this case the errors were $-0.04^{\circ}$ and $-0.14^{\circ}$ for the pitch and roll angles.

For image 8533 in full resolution, the error mode is the same as for image 8913, with a long lake edge inside the true horizon. But, in addition, haze in the image conceals and denies the detection of an edge for the true horizon in quite a large part of the image. In total, this means that when extracting horizon edge pixels, a very large fraction of lake edges are included generating a total angular error around $0.6^{\circ}$.

New camera calibration. For high resolution images (full resolution and downscaled a factor 2), the overall results could be further improved by including some images from the southbound part of the flight in the set of images used for camera calibration. The results are listed in table 5 and are illustrated in figure 17. The total error is now almost equal over the full flight. Still there is a small jump in the mean level for both attitude angle errors when turning from a northbound to a southbound flight. The cause of this mean level jump is probably the Vättern effect and haze pushing the detected horizon in the image slightly inside the true horizon. This behavior will affect the camera calibration and the attitude estimation results. Still, the attitude estimate errors achieved using the proposed method are less or equal to previous methods with DEM refinement and far better than for any method without DEM refinement.

Table 5: Attitude estimation errors in degrees after DEM refinement. Calibration with images from northbound and southbound parts of flight.

\begin{tabular}{|c|c|c|c|c|c|c|c|}
\hline $\begin{array}{c}\text { Scale } \\
\text { factor }\end{array}$ & $\begin{array}{c}\text { Image } \\
\text { size }\end{array}$ & $\begin{array}{c}\text { Pitch } \\
\text { mean }\end{array}$ & $\begin{array}{c}\text { Pitch } \\
\text { std }\end{array}$ & $\begin{array}{c}\text { Roll } \\
\text { mean }\end{array}$ & $\begin{array}{c}\text { Roll } \\
\text { std }\end{array}$ & $\begin{array}{c}\text { Total } \\
\text { mean }\end{array}$ & $\begin{array}{c}\text { Total } \\
\text { std }\end{array}$ \\
\hline 2 & $3 \mathrm{~K} \times 2.5 \mathrm{~K}$ & 0.007 & 0.038 & -0.001 & 0.046 & 0.054 & 0.029 \\
Full & $6 \mathrm{~K} \times 5 \mathrm{~K}$ & 0.017 & 0.035 & 0.003 & 0.044 & 0.050 & 0.030 \\
\hline
\end{tabular}

\subsubsection{Results with uncertain altitude, position and heading}

The results after DEM refinement presented above have assumed that we have perfect knowledge of the aircraft 3D position and heading angle when computing the geometric horizon. In reality these parameters will be known with a certain error. To determine how sensitive the DEM refinement is to these errors, attitude estimation runs have been made where the assumed 3D position and heading angle were randomised with reasonable errors. Uniform distributions around the true value were used with the following errors; altitude: $\pm 10 \%, \mathrm{X}$ and $\mathrm{Y}$ positions: $\pm 50 \mathrm{~m}$, heading angle: $\pm 5^{\circ}$. The attitude estimation results for full resolution images with and without the initialization errors are listed in table 6 . The results show that the accuracy of the attitude estimate is reduced but the degradation is small.

Table 6: Attitude estimation errors in degrees with and without initialization errors on altitude, XY position and heading angle.

\begin{tabular}{|c|c|c|c|c|c|c|c|c|}
\hline Errors & $\begin{array}{c}\text { Scale } \\
\text { factor }\end{array}$ & $\begin{array}{c}\text { Image } \\
\text { size }\end{array}$ & $\begin{array}{c}\text { Pitch } \\
\text { mean }\end{array}$ & $\begin{array}{c}\text { Pitch } \\
\text { std }\end{array}$ & $\begin{array}{c}\text { Roll } \\
\text { mean }\end{array}$ & $\begin{array}{c}\text { Roll } \\
\text { std }\end{array}$ & $\begin{array}{c}\text { Total } \\
\text { mean }\end{array}$ & $\begin{array}{c}\text { Total } \\
\text { std }\end{array}$ \\
\hline No errors & Full & $6 \mathrm{~K} \times 5 \mathrm{~K}$ & 0.017 & 0.035 & 0.003 & 0.044 & 0.050 & 0.030 \\
Init. errors & Full & $6 \mathrm{~K} \times 5 \mathrm{~K}$ & 0.016 & 0.039 & 0.001 & 0.050 & 0.055 & 0.034 \\
\hline
\end{tabular}




\section{Downscaled a factor 2}
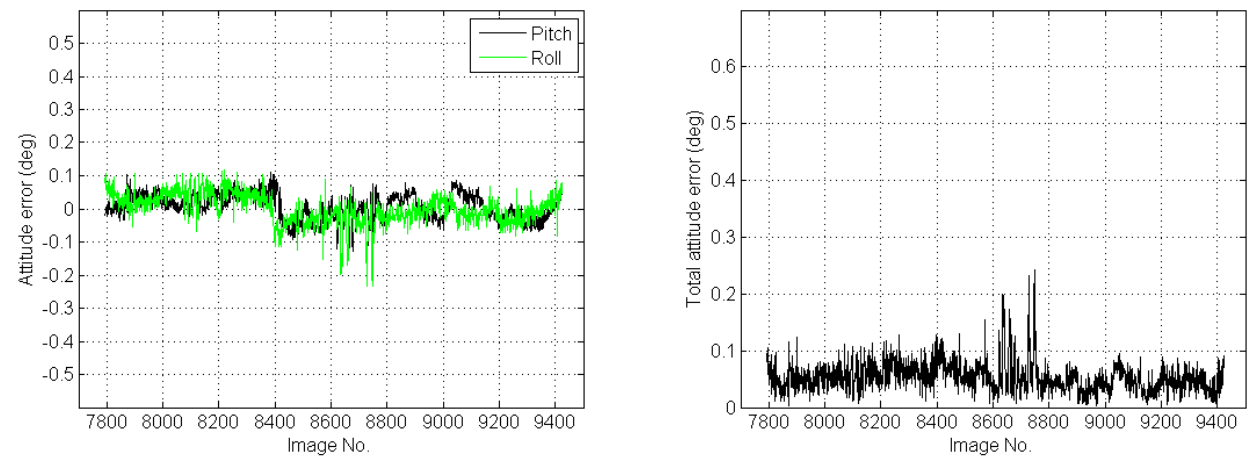

Full resolution
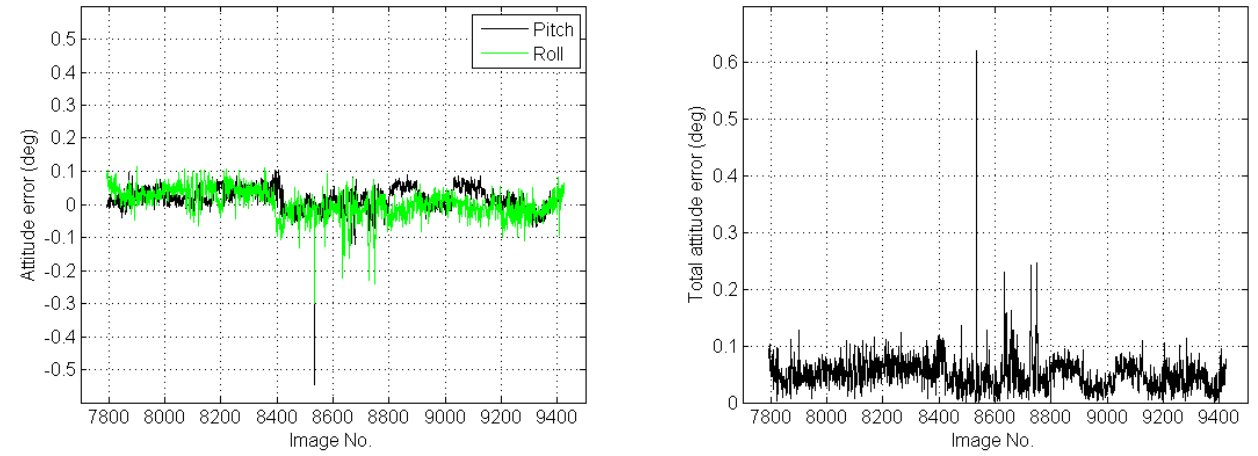

Figure 17: Results after DEM refinement with new camera calibration. Images downscaled a factor 2 and full resolution.

\subsubsection{Results without refracted rays}

One of the key components claimed to be required to achieve the high accuracy attitude estimates is taking the refraction of light rays in the earth atmosphere into account. How crucial is this factor? What accuracy can be obtained if neglecting the refraction, i.e. if assuming a constant index of refraction in the atmosphere. To investigate this, runs have been carried out with the following assumptions. The camera calibration as computed from refracted rays was used since this calibration result could have been obtained in lab environment with an appropriate calibration object. The incidence angle on the unit sphere as a function of camera altitude was recomputed assuming nonrefracted (straight) rays. The LUTs for the incidence angle on the unit sphere for object heights at various distances from the camera were recomputed assuming nonrefracted rays.

The results are listed in table 7 and are illustrated in figure 18. For images captured during the northbound part of the flight, up to image 8400 , there is a clear degradation of the results. The mean error for both the pitch and roll angles is considerably larger compared to when ray refraction is taken into account. For straight rays, the distance to the horizon is shorter and the incidence angle on the unit sphere is smaller. This means that the geometrically expected ellipse on the image plane will be smaller. To compensate for the true larger ellipse on the image plane, the aircraft pitch and roll angles need to be increased thus inducing larger errors. The same reasoning holds for the southbound part of the flight as well. Here, the Vättern effect conceals some of the degradation but the trend with larger mean errors on the attitude angles remains. 
Table 7: Attitude estimation errors in degrees with and without refracted rays.

\begin{tabular}{|c|c|c|c|c|c|c|c|c|}
\hline Rays & $\begin{array}{c}\text { Scale } \\
\text { factor }\end{array}$ & $\begin{array}{c}\text { Image } \\
\text { size }\end{array}$ & $\begin{array}{c}\text { Pitch } \\
\text { mean }\end{array}$ & $\begin{array}{c}\text { Pitch } \\
\text { std }\end{array}$ & $\begin{array}{c}\text { Roll } \\
\text { mean }\end{array}$ & $\begin{array}{c}\text { Roll } \\
\text { std }\end{array}$ & $\begin{array}{c}\text { Total } \\
\text { mean }\end{array}$ & $\begin{array}{c}\text { Total } \\
\text { std }\end{array}$ \\
\hline Refracted & Full & $6 \mathrm{~K} \times 5 \mathrm{~K}$ & 0.017 & 0.035 & 0.003 & 0.044 & 0.050 & 0.030 \\
Straight & Full & $6 \mathrm{~K} \times 5 \mathrm{~K}$ & 0.049 & 0.036 & 0.040 & 0.053 & 0.085 & 0.034 \\
\hline
\end{tabular}
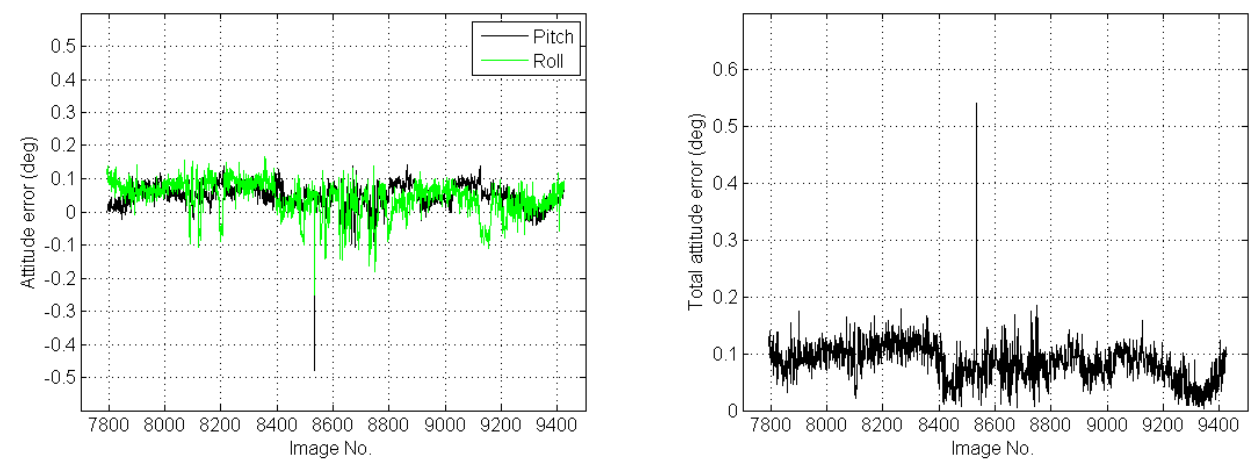

Figure 18: Results after DEM refinement assuming straight rays.

\subsubsection{Error analysis}

The results presented above have shown that after DEM refinement the mean error for the estimated pitch and roll angles is smaller than the pixel resolution (which is $0.04^{\circ}$ ) and the standard deviation for the errors is in the same order as the pixel resolution. Is the accuracy obtained quantitatively reasonable and what are the factors determining the accuracy? The main error sources in our flight trial are explained below and summarized in table 8.

Table 8: Error sources and their impact on the attitude estimates.

\begin{tabular}{|l|c|l|}
\hline Error source & Error $(\mathrm{deg})$ & Comments \\
\hline Ground truth accuracy & 0.01 & \\
DEM resolution and accuracy & $\sim 0.01$ & Larger errors in hilly terrain \\
Ray refraction & $<0.01$ & Offset errors $0.05^{\circ}-0.10^{\circ}$ if ray refraction is ignored \\
Image scene content & $0-0.2$ & Often close to zero, in rare cases (lake edges) up to $0.2^{\circ}$ \\
Camera calibration & $0.01-0.02$ & Depend on ground truth and DEM accuracy \\
\hline
\end{tabular}

Ground truth accuracy. The accuracy of the ground truth orientation from the navigation sensors is claimed to be around $0.01^{\circ}$ for all attitude angles. The uncertainty measure for the filtered solution varies during flight depending on the excitation of the gyros and IMUs. As previously stated, the navigation sensors were placed inside cock-pit whereas the camera was mounted below the aircraft. This means that the navigation sensors and the camera may not experience exactly the same forces and their relative orientation may differ slightly during flight. This may be one of the reasons that the largest errors for the attitude estimates coincide with large aircraft maneuvers.

DEM resolution and accuracy. The DEM used to compute the geometrically expected horizon is the SRTM3 database where elevations are given for a 90x90 m grid on the ground. The SRTM3 data was generated from satellite radar measurements. The radar measures an average of the altitude within an XY grid cell, and it also measures an average between reflections from the ground and overlaying structures as roofs or treetops. This is not ideal when computing a geometrical horizon from an airborne visual sensor. It would be more appropriate to use a DEM containing the maximum elevation within each cell since this is what a visual sensor will perceive as the horizon. However, this type of DEM is rarely available with large area coverage. The standard deviation for the SRTM3 elevations is claimed to be around $8 \mathrm{~m}$. Due to 
elevation variations within the grid cell, the difference between the maximum and the mean elevations can easily be around $20 \mathrm{~m}$ in hilly terrain whereas the two values coincide for level ground. To illustrate the impact of this elevation uncertainty on the attitude estimate we use a numerical example. For an aircraft flying at $400 \mathrm{~m}$ altitude, changing the ground elevation at the horizon from $200 \mathrm{~m}$ to $220 \mathrm{~m}$ generates a shift of the perceived horizon that is close to $0.01^{\circ}$.

Ray refraction. In section 7.3.4 it was shown how the accuracy of the attitude estimates was degraded if ray refraction in the atmosphere was not taken into account. A spherically stratified model for the variation of the refractive index with altitude was used. The model assumes that the air pressure and temperature varies with the altitude above the ground in a standardized manner. This is a first order approximation and does not take into account local variations in air pressure and temperature. However, since the difference between refracted rays and straight rays generated an attitude estimate shift around $0.05^{\circ}$, it seems reasonable that the deviation from the first order approximation generates errors that are smaller than $0.01^{\circ}$.

Image scene content. Depending on the scene, this may be the largest source of error. A typical example is the Vättern effect where lake edges in the image interfere strongly with the detection of the true horizon. Lake edges, or edges from other objects in the scene, may conceal or merge with the true horizon in the image. We are then dealing with local shifts in the perceived horizon that amounts to several pixels. For our full image resolution this translates to errors in the order of $0.1-0.2^{\circ}$.

Weather phenomena like presence of haze or fog may also induce errors on the horizon detection, and in turn on the attitude estimates. In our flight there was haze when looking in the west-northwest direction. This makes the perceived horizon more diffuse in the images and it may shift the location of the horizon edge in the Canny detector slightly. The effect of haze is more pronounced at higher altitudes since then the light rays need to propagate a longer distance through air to reach the horizon. We have no quantitative estimate of the errors induced by haze on the attitude estimates.

Camera calibration. In our case we used ground truth orientation data and elevation data from the DEM to refine the camera calibration, both the intrinsic parameters and the camera mounting on the aircraft frame. Even if we carefully select images where the image scene content does not introduce erroneous edges, the errors from the ground truth and the DEM combined accumulate to 0.01-0.02 . These errors will inevitably generate small errors on the camera calibration.

The camera calibration includes a nonlinear optimization to find a total of 14 intrinsic and extrinsic camera parameters. It was evident that different camera calibrations were obtained depending on the image set used for calibration and also the initialization values in the nonlinear optimization. However, evaluations showed that very similar overall attitude estimation results were obtained for slightly different camera calibrations.

Total error. When adding the errors from the camera calibration and the DEM, error propagation will lead to total attitude estimate errors in the order $0.02-0.03^{\circ}$. This is also very close to the accuracy that was actually achieved with the proposed method for the full resolution images. The somewhat larger errors that were obtained can probably be attributed to the images where the Vättern effect was evident. To further improve the results, either a more accurate camera calibration method or a DEM with finer grid cell size and higher elevation accuracy than provided by SRTM3 is required. The error analysis also explains why the attitude estimates are not significantly better for the full resolution images compared with images downscaled a factor 2 , c.f. table 5 .

\subsection{Implementation and processing times}

The first four algorithmic steps in our method, c.f. figure 4, are coded in $\mathrm{C}++$, where the OpenCV implementations of the Canny detector and the Hough circle detector have been used as a base. The last two steps in the algorithm as well as the camera calibration refinement are written in Matlab. Processing times for different image scales are listed in table 9. The processing times reported are valid for a standard laptop, 
Table 9: Processing times. Canny detector and Hough voting for complete image and for $\pm 2^{\circ}$ band around coarse horizon estimate. Attitude refinement including and excluding computation of geometrical horizon.

\begin{tabular}{|c|c|c|c|c|c|}
\hline $\begin{array}{c}\text { Scale } \\
\text { factor }\end{array}$ & $\begin{array}{c}\text { Image } \\
\text { size }\end{array}$ & $\begin{array}{c}\text { Canny + Hough } \\
\text { Complete image }\end{array}$ & $\begin{array}{c}\text { Canny + Hough } \\
\text { Horizon band }\end{array}$ & $\begin{array}{c}\text { Refinement } \\
\text { incl. horizon }\end{array}$ & $\begin{array}{c}\text { Refinement } \\
\text { excl. horizon }\end{array}$ \\
\hline 8 & $0.8 \mathrm{Kx} 0.6 \mathrm{~K}$ & $0.1-0.4 \mathrm{~s}$ & $0.1-0.4 \mathrm{~s}$ & $0.4 \mathrm{~s}$ & $15-20 \mathrm{~ms}$ \\
4 & $1.5 \mathrm{Kx} 1.2 \mathrm{~K}$ & $0.5-1,5 \mathrm{~s}$ & $0.2-0.6 \mathrm{~s}$ & $0.8 \mathrm{~s}$ & $25-30 \mathrm{~ms}$ \\
2 & $3 \mathrm{~K} 2.5 \mathrm{~K}$ & $1.5-5 \mathrm{~s}$ & $0.3-0.8 \mathrm{~s}$ & $1.5 \mathrm{~s}$ & $40-50 \mathrm{~ms}$ \\
Full & $6 \mathrm{~K} 5 \mathrm{~K}$ & $5-20 \mathrm{~s}$ & $0.4-1.2 \mathrm{~s}$ & $3.0 \mathrm{~s}$ & $80-100 \mathrm{~ms}$ \\
\hline
\end{tabular}

an Intel Core i5 CPU M560 @ 2.67 GHz.

The processing times for the Canny detector and the Hough voting are strongly dependent on the image scene content. For the full resolution image, the Canny detector and the Hough voting take 5-20 s if edge pixels over the complete image are processed. To reduce the processing time, we first compute a coarse attitude estimate at low image resolution (images downscaled a factor 8). For this image resolution, the processing time for the Canny detector and the Hough voting is 0.1-0.4 s. For higher image resolutions, we only evaluate pixels within a $\pm 2^{\circ}$ wide band around the coarse attitude estimate. This reduces the processing time for the Canny detector and Hough voting to $0.4-1.2 \mathrm{~s}$ for the full resolution image. Using this scheme, the total processing time increases almost linearly with the image resolution.

As an average over all images, the attitude refinement takes about $0.4 \mathrm{~s}$ for images downscaled a factor 8 and about $3.0 \mathrm{~s}$ for the full resolution image. The major part $(\sim 95 \%)$ of the refinement processing time is the computation of the geometric horizon. If we use the same concept as in previous work (Dumble and Gibbens, 2014) and pre-compute the geometric horizon, the refinement processing time is $80-100 \mathrm{~ms}$ for the full resolution image.

Assuming a pre-computed geometric horizon, the total processing time for the full resolution image with the current implementation is on average around $1.5 \mathrm{~s}$. The predominant time factor is the Hough voting which is readily parallelized. The above processing times indicate that with a multi-threaded $\mathrm{C}++$ implementation of the complete attitude estimation algorithm, the total processing time would be far less than $1 \mathrm{~s}$ for the full resolution image.

\subsection{Comparison of methods and results}

As mentioned in the related work, section 2, we have found only one previous method for aerial images where the attitude estimates are refined by registration of the perceived horizon with a geometric horizon computed from a DEM (Dumble and Gibbens, 2014). This section is intended to describe the differences and the similarities between that method and ours. The main characteristics of the two methods are given in table 10.

Table 10: Comparison between the previous method (Dumble and Gibbens, 2014) and our method.

\begin{tabular}{|l|l|l|}
\hline Characteristic & Dumble and Gibbens 2014 & Our method \\
\hline Accuracy pitch and roll angles & $\sigma=0.07^{\circ}$ and $0.03^{\circ}$ & $\sigma=0.04^{\circ}$ and $0.05^{\circ}$ \\
Terrain type best suited for & Mountainous & Level and rolling \\
Evaluation images & Synthetic images & Real images \\
Estimated entities & Attitude and refined location & Attitude \\
DEM & Flight simulator used for image rendering & SRTM3 \\
Accounts for ray refraction & No & Yes \\
Camera type & Four perspective cameras & Fisheye camera \\
Image size & $1024 x 768$ pixels (x 4 images) & $6144 x 4912$ pixels \\
Execution time & $\sim 50 \mathrm{~ms}$ & $\sim 1 \mathrm{~s}$ \\
\hline
\end{tabular}


One major difference between the two seemingly similar methods is that they are suited for very different types of terrain. The previous method (Dumble and Gibbens, 2014) requires a large horizon profile variance which is definitely fulfilled in the Alps where their virtual evaluation flight was conducted. In their paper, they show that their method degrades when lowering the horizon profile variance and also that it becomes unstable for variances lower than $1^{0^{2}}$, figure 11 in (Dumble and Gibbens, 2014). In our flight, conducted in level and rolling terrain, the horizon profile variance varied between $0.001-0.007^{\circ}$, i.e. the variance is far too small for their method to be stable. Contrary, a small horizon profile variance is advantageous for our method since we search for true horizon pixels in a thin band close to the ideal horizon at sea level.

Another evidence that their method is suited for mountainous terrain is a statement in their paper that their method fails to detect a distant horizon. "At the start and end of the simulation, the backwards and then the forwards facing camera respectively are facing the distant terrain $(+40 \mathrm{~km})$. In these situations the horizon detection algorithm cannot detect the distant horizon, so the detected profile sits lower in the image than where the distant terrain would be." The distance $40 \mathrm{~km}$ to the ideal horizon at sea level corresponds to a flight altitude around $100 \mathrm{~m}$. In our flight, in level and rolling terrain, the detected horizon was further away than $40 \mathrm{~km}$ in more than $99 \%$ of the horizon pixels. Under these circumstances, their method is unlikely to achieve accurate results.

Both methods rely on measuring more than half of the complete horizon line. Their method uses four perspective cameras whereas we use one fisheye camera. There are two main differences between these two camera solutions. First, camera calibration of the intrinsic parameters is considerably easier for a perspective camera than for a fisheye camera. On the other hand, in reality, there will be alignment errors between the four perspective cameras. This error source has been neglected in their paper since they rendered synthetic images from ideal camera positions. Second, the detection of the true horizon line in the image is completely different. For the perspective camera, they search for a continuous line over the whole image to suppress other edges. This makes their method vulnerable to partial occlusion of the horizon due to e.g. haze, fog and clouds. For the fisheye image, we search for the circle on the unit sphere (ellipse on the image plane) with the strongest support from all edge pixels. Our method can cope with partial occlusion of the horizon line caused by various weather phenomena.

The different strategies for detecting the horizon in the image for the two methods prevent the same set of images to be used to compare the two methods. In their method, they search for one horizon edge pixel along each column of a perspective image and this strategy cannot be transferred to an omnidirectional image where the horizon is an ellipse on the image plane. Conceivably, we could modify our method to take edge pixels and the corresponding edge directions from a set of perspective images around the aircraft as input to the Hough voting. But, taking perspective images as input would require a change to the existing system and would evaluate some other method than the proposed.

Both methods give roughly the same error on the attitude estimates. The errors obtained are about one order of magnitude smaller than for any other method without DEM refinement. However, we need to emphasize that our results were obtained using real images whereas their evaluation was based on synthetic images. Presumably, they used the same flight simulation program to compute the geometric horizon as they used to render the images, i.e. there is effectively no geometric discrepancy between the elevation model and the world appearance, thus the DEM error source is ignored. Furthermore, ray refraction in the atmosphere is not taken into account in their model. In our flight, this induced a systematic attitude error in the order of $0.05^{\circ}$, depending on the flight altitude and ground elevations in the surrounding terrain.

Both methods require an approximate 3D position in a world coordinate frame as input. This 3D position could be obtained from a GPS or, if GPS free navigation is mandatory, employing a vision-based terrain navigation method. In the previous method (Dumble and Gibbens, 2014), the position is used to select the closest pre-computed horizon profile which, in turn, is used for estimating the attitude and refining the position estimate through alignment with the horizon profile in the image. In our method, the approximate $3 \mathrm{D}$ position is used for computing the geometrical horizon and we do not refine the position estimate. In a low variance horizon profile environment, the pitch and roll angles are the only two parameters in the 
complete aircraft $6 \mathrm{DOF}$ pose that can always be determined. They can be determined even if the horizon would be smooth and perfectly level. This is the reason why we only estimate the pitch and roll angles in the proposed method and why we rely on complementary methods, e.g. (Grelsson et al., 2013), to refine the position and heading estimates.

Obviuosly, their method is faster than ours. If we assume that the geometric horizon is pre-computed, their method runs at about $20 \mathrm{~Hz}$ and ours at $1 \mathrm{~Hz}$. The main reason for the difference in speed is that we use images with nearly 10 times more pixels. Still, most important is that both methods provide absolute attitude measurements. When used in a filtering network together with an IMU, the absolute attitude measurements can bound the drift in the IMU and also assist in vertical alignment calibration. Today there is a large variety of industrial grade or higher MEMS IMUs with drift less than $30^{\circ} / \mathrm{h}$ (or $0.01^{\circ} / \mathrm{s}$ ). For these IMUs, a measurement rate of $1 \mathrm{~Hz}$ for complementary absolute attitude measurements is sufficient to support online vehicle navigation, although a higher rate is of course preferable if the same accuracy can be provided.

Both methods can also be used as stand-alone sensors for attitude estimation. The auto correlation for the pitch and roll errors obtained with our method is 0.4-0.5. We expect that filtering the results of our estimation method will further improve the results and experiments to verify this will be subject of future work.

\subsection{Discussion and future work}

In this section we discuss some potential modifications and future improvements to our proposed method.

The camera used in the flight trial had a FOV of $183^{\circ}$ implying that for some tilt angles the complete horizon around the aircraft could potentially be seen. However, for ease of mounting the camera on the aircraft, as mentioned in section 6 , the optical axis of the camera was not vertical when the aircraft was in level flight thus preventing the complete horizon to be seen. In fact, for most of the images from our test flight about half of the complete horizon could be seen and in just a handful of images, depending on the tilt angle, up to $2 / 3$ of the full horizon was seen. No difference in the attitude accuracy could be noted for these images in comparison but they were too few to give a statistically significant statement.

Intuitively, it would be favorable for the attitude estimates to see the complete horizon in the image mainly for robustness reasons. When the geometry allows the complete horizon to be seen it is more likely that the Hough voting will give a good initial attitude estimate if there are lake edges close to the true horizon or if partial occlusion of the horizon occurs due to haze. In addition, the refined attitude estimate ought to be more accurate if there are more data points used for the alignment with the geometrical horizon. Our test flight contains too few images available to support the suggested improvement of robustness and accuracy if a larger portion of the horizon is seen. However, the reverse could be noted. Many of the large attitude estimate errors occurred when haze prevented a large portion of the horizon to be seen and at the same time there were lake edges present close to the horizon. Therefore, we recommend mounting the camera such that the geometry allows the complete horizon to be seen for the nominal aircraft pitch angle during flight.

Concerning flights in mountainous terrain with large horizon profile variance, we cannot foresee any fundamental limitation in our method from working in such an environment. The initial attitude estimate from the Hough voting would result in a too small ellipse on the image plane falling inside the true horizon profile. With a wide band around the ideal horizon as indicated by the horizon profile from the DEM, the true horizon edge pixels would be extracted enabling a good alignment between the horizon in the image and the geometrical horizon. If flying in mountainous terrain we would also need to include ground objects, within the distance to the ideal horizon, at higher altitudes than the camera in the LUTs. The reason that we only

have images from level and rolling terrain in our evaluation is that there are no mountains in the vicinity of our location in Linköping, Sweden, as in the majority of other places. 
In the proposed method, we estimate the pitch and the roll angle. It would be rather straightforward to modify our method to also refine the yaw estimate provided that the horizon profile variance, as indicated by the DEM, is sufficiently large. The minimization in (20) would then be made over all three euler angles, now it is made over the pitch and the roll angle. If the horizon profile variance is large, it would probably be favorable to slightly modify the method for extraction of horizon edge pixels. Currently we search for horizon edge pixels in a thin band with equal width around the ideal horizon. The width and position of this band could be adapted for different azimuth angles around the unit sphere in accordance with the geometrical horizon profile computed from the DEM, provided that an approximate heading angle is known. This modification would reduce the risk of extracting edge pixels from lakes and clouds as horizon edges and prevent them for contributing to errors in the final attitude refinement.

We have some ideas how to mitigate the Vättern effect. In the DEM, lakes ant other flat regions could readily be detected and labelled. When computing the geometrical horizon, we could also search for expected lake (flat region) edges close to the horizon. If, geometrically, there is a lake edge that cannot be resolved from the horizon in the DEM for a certain azimuth angle, given the camera resolution used, we would ignore that direction in the attitude refinement. In other words, we would introduce weights in the error function (19) and the weights would be set to zero in those directions where the lake edge is not expected to be resolved from the true horizon. Potentially, this could lead to a refined attitude estimate being based on a relatively small portion of the horizon, implying a less robust estimate. This could be compensated for by lowering the confidence in the attitude estimate, i.e. weighting the covariance matrix based on the number of edge pixels used in the refinement process.

\section{Conclusions}

We have presented a novel method for highly accurate estimation of the pitch and roll angles of an airborne vehicle by horizon detection in fisheye images. A Canny edge detector and a probabilistic Hough voting scheme are used to compute an approximate vehicle attitude and the corresponding horizon line in the image. Horizon edge pixels are extracted in a thin band in the vicinity of the approximate horizon line. The attitude estimates are refined through registration of the extracted horizon edge pixels with the geometrical horizon from a DEM. To the best of our knowledge, this is the first proposed method for aerial omnidirectional images where the detected horizon is refined with DEM data for attitude estimation.

The method has been evaluated with a flight trial where 1629 images were captured at flight altitudes up to $600 \mathrm{~m}$ in an area with ground elevations ranging from sea level up to $500 \mathrm{~m}$. The vision-based pitch and roll angle estimates were compared with the ground truth from navigation sensors, a filtered IMU/GPS solution. For the full resolution images, the standard deviation for the pitch and roll angle errors are $0.04^{\circ}$ and $0.05^{\circ}$, respectively, with mean errors smaller than $0.02^{\circ}$. The attitude errors obtained on real images are less or equal to those achieved on synthetic images for previous methods with DEM refinement, and the errors are about one order of magnitude smaller than for any previous vision-based method without DEM refinement. To achieve the high accuracy attitude estimates, the ray refraction in the earth atmosphere has been taken into account.

One error source for the proposed method is the presence of edges generated by image scene content in the vicinity of the true horizon line. When these edges are extracted as horizon edge pixels, they will induce errors in the refinement step when registering the horizon edge pixels with DEM data. Although long lake edges were present in a large fraction of the images from our flight, the errors in the estimated pitch and roll angles were still rarely larger than $0.1^{\circ}$.

\section{Acknowledgements}

This work was funded by the Swedish Governmental Agency for Innovation Systems, VINNOVA, under contract NFFP5 2013-05243, and supported by the Swedish Foundation for Strategic Research through 
grant RIT10-0047 (CUAS). This research has received funding from the Swedish Research Council through a grant for the project Extended Target Tracking (within the Linnaeus environment CADICS).

\section{References}

Active Vision Group, University of Oxford (2013). http://www.robots.ox.ac.uk/ cmei/Toolbox.html.

Applanix (2014). http://applanix.com/media/downloads/products/specs/posav_specs_1212.pdf.

Baatz, G., Saurer, O., Köser, K., and Pollefeys, M. (2012). Large Scale Visual Geo-Localization of Images in Mountainous Terrain. ECCV.

Bao, G., Zhou, Z., Xiong, S., Lin, X., and Ye, X. (2003). Towards Micro Air Vehicle Flight Autonomy Research on The Method of Horizon Extraction. IMTC.

Bazin, J.-C., I. Kweon, C. D., and Vasseur, P. (2008). UAV attitude estimation by vanishing points in catadioptric images. IEEE International Conference on Robotics and Automation, pages 2743-2749.

Bazin, J.-C., I. Kweon, C. D., and Vasseur, P. (2009). Dynamic Programming and Skyline Extraction in Catadioptric Infrared Images. IEEE International Conference on Robotics and Automation.

Behringer, R. (1998). Improving the Registration Precision by Visual Horizon Silhouette Matching. Proc. of the First IEEE Workshop on Augmented Reality.

Canny, J. (1986). A computational approach to edge detection. PAMI, 8:679-698.

Demonceaux, C., Vasseur, P., and Pégard, C. (2007). UAV attitude computation by omnidirectional vision in urban environment. International Conference on Robotics and Automation, pages 2017-2022.

Dumble, S. J. and Gibbens, P. W. (2012). Horizon Profile Detection for Attitude Estimation. Journal of Intelligent Robotic Systems, 68:339-357.

Dumble, S. J. and Gibbens, P. W. (2014). Efficient Terrain-Aided Visual Horizon Based Attitude Estimation and Localization. Journal of Intelligent Robotic Systems.

Dusha, D., Mejias, L., and Walker, R. (2011). Fixed-Wing Attitude Estimation Using Temporal Tracking of the Horizon and Optical Flow. Journal of Field Robotics, 28(3):355-372.

Encyclopaedia Britannica (2014). http://www.britannica.com/EBchecked/media/125692/Hypsographiccurve-showing-how-the-surface-area-of-Earth-is.

Grelsson, B. and Felsberg, M. (2013). Probabilistic Hough Voting for Attitude Estimation from Aerial Fisheye Images. 18th Scandinavian Conference in Image Analysis, SCIA 2013, pages 478-488.

Grelsson, B., Felsberg, M., and Isaksson, F. (2013). Efficient 7D Aerial Pose Estimation. IEEE Workshop on Robot Vision.

Groves, P. D. (2013). Principles of GNSS, inertial, and multisensor integrated navigation systems. Artech House.

Gupta, V. and Brennan, S. (2008). Terrain-Based Vehicle Orientation Estimation Combining Vision and Inertial Measurements. Journal of Field Robotics, 25(3):181-202.

Gyer, M. (1996). Methods for Computing Photogrammetrix Refraction Corrections for Vertical and Oblique Photographs. Photogrammetric Engineering and Remote Sensing, 62(3):301-310.

Hough, P. (1962). Method and means for recogninzing complex patterns. U.S. Patent 3069654.

Mondragon, I. F., Campoy, P., Martinez, C., and Olivares, M. (2010). Omnidirectional Vision applied to Unmanned Aerial Vehicles (UAVs) attitude and heading estimation. Robotics and Autonomous Systems, 58(6):809-919. 
Moore, R. J. D., Thurrowgood, S., Bland, D., Soccol, D., and Srinivasan, M. V. (2011a). A Fast and Adaptive Method for Estimating UAV Attitude from the Visual Horizon. Proc. IEEE International Conference on Intelligent Robots and Systems.

Moore, R. J. D., Thurrowgood, S., Soccol, D., Bland, D., and Srinivasan, M. V. (2011b). A Method for the Visual Estimation and Control of 3-DOF Attitude for UAVs. Proc. ARAA Australasian Conference on Robotics and Automation.

NASA (2013). http://www2.jpl.nasa.gov/srtm/.

National Physical Laboratory (2013). http://www.kayelaby.npl.co.uk/general_physics/.

Natraj, A., Ly, D. S., Eynard, D., Demonceaux, C., and Vasseur, P. (2013). Omnidirectional Vision for UAV: Applications to Attitude, Motion and Altitude Estimation for Day and Night Conditions. Journal of Intelligent Robotic Systems, 69:459-473.

Naval Jr., P. C., Mukunoki, M., Minoh, M., and Ikeda, K. (1997). Estimating Camera Position and Orientation from Geographical Map and Mountain Image. 38th Research Meeting of the Pattern Sensing Group, Society of Instrument and Control Engineers.

NewGeography (2010). http://www.newgeography.com/content/001689-how-much-world-covered-cities.

Shabayek, A. E. R., Demonceaux, C., Morel, O., and Fofi, D. (2012). Vison Based UAV Attitude Estimation: Progress and Insights. Journal of Intelligent Robot Systems.

Thurrowgood, S., Moore, R. J. D., Bland, D., Soccol, D., and Srinivasan, M. V. (2010). UAV Attitude Control using the Visual Horizon. Twelfth Australasian Conference on Robotics and Automation (ARCA 2010).

Woo, J., Son, K., Li, T., Kim, G., and Kweon, I. S. (2007). Vision-based UAV Navigation in Mountain Area. IAPR Conference on Machine Vision Applications, pages 236-239. 